\title{
Quasiparticle random-phase approximation with quasiparticle-vibration coupling: Application to the Gamow-Teller response of the superfluid nucleus ${ }^{120} \mathrm{Sn}$
}

\author{
Y. F. Niu (牛一斐), ${ }^{1,2, *}$ G. Colò, ${ }^{1,3}$ E. Vigezzi, ${ }^{1}$ C. L. Bai (白春林), ${ }^{4}$ and H. Sagawa ${ }^{5,6}$ \\ ${ }^{1}$ INFN, Sezione di Milano, Via Celoria 16, I-20133 Milano, Italy \\ ${ }^{2}$ ELI-NP, "Horia Hulubei” National Institute for Physics and Nuclear Engineering, 30 Reactorului Street, \\ RO-077125 Bucharest-Magurele, Romania \\ ${ }^{3}$ Dipartimento di Fisica, Università degli Studi di Milano, Via Celoria 16, I-20133 Milano, Italy \\ ${ }^{4}$ School of Physical Science and Technology, Sichuan University, Chengdu 610065, China \\ ${ }^{5}$ RIKEN, Nishina Center, Wako 351-0198, Japan \\ ${ }^{6}$ Center for Mathematics and Physics, University of Aizu, Aizu-Wakamatsu, Fukushima 965-8580, Japan \\ (Received 8 September 2016; revised manuscript received 20 November 2016; published 29 December 2016)
}

\begin{abstract}
We propose a self-consistent quasiparticle random-phase approximation (QRPA) plus quasiparticle-vibration coupling (QPVC) model with Skyrme interactions to describe the width and the line shape of giant resonances in open-shell nuclei, in which the effect of superfluidity should be taken into account in both the ground state and the excited states. We apply the new model to the Gamow-Teller resonance in the superfluid nucleus ${ }^{120} \mathrm{Sn}$, including both the isoscalar spin-triplet and the isovector spin-singlet pairing interactions. The strength distribution in ${ }^{120} \mathrm{Sn}$ is well reproduced and the underlying microscopic mechanisms, related to QPVC and also to isoscalar pairing, are analyzed in detail.
\end{abstract}

DOI: 10.1103/PhysRevC.94.064328

\section{INTRODUCTION}

Nuclear charge-exchange transitions correspond to the transitions from an initial state in the nucleus $(N, Z)$ to the final states in the neighboring nuclei $(N+1, Z-1)$ or $(N-$ $1, Z+1)[1,2]$. Among the most widely known transitions are the isobaric analog resonance (IAR), the Gamow-Teller resonance (GTR), and the spin-dipole resonance (SDR). These different vibrational modes, that involve spin and isospin degrees of freedom, provide direct and valuable information on the isospin- and spin-isospin-dependent parts of the effective interaction in the nuclear medium, which are otherwise poorly constrained. Nuclear charge-exchange transitions play also a crucial role in nuclear astrophysics. GT excitations are the dominant excitation modes in weak-interaction processes such as $\beta$ decay, electron capture, and neutrino-nucleus reactions $[3,4]$. $\beta$-decay half-lives set the time scale of the $r$ process, and hence determine the production of heavy elements in the universe [5-7]. Electron capture governs the evolution of massive stars at the end of their last hydrostatic burning phase, and influences the dynamics of core-collapse supernovae [3,4,8-10]. A very accurate knowledge of spin-isospin matrix elements is also instrumental to extract the properties of neutrinos from the measured half-life of double- $\beta$ decay $[11,12]$. Therefore, nuclear charge-exchange transitions capture the interests of researchers, both experimentalists and theorists, not only in nuclear physics but also in particle physics and astrophysics.

Whereas nuclear $\beta$ decay provides directly the values of the nuclear matrix elements of the relevant transition operator, this is not the case when the charge-exchange states are populated by charge-exchange reactions such as $(p, n)$ or $\left({ }^{3} \mathrm{He}, t\right)$. The proportionality between the reaction cross sections at the

*nyfster@gmail.com forward angles and the GT strength has been proven to a large extent, especially for strong GT transitions, and this has paved the way to a direct extraction of the GT matrix elements from reaction measurements. Yet this procedure is not entirely free from ambiguities. Moreover, no clear proportionality has been established in the case of higher multipoles. In such a situation, it is of paramount importance to try to improve the predictive power of theoretical models that can provide directly the transition strengths of the charge-exchange states of interest throughout the nuclear chart.

Two types of microscopic approaches are widely used in the theoretical investigation of the charge-exchange excitations: i.e., the shell model and the random-phase approximation (RPA) approach, which becomes quasiparticle RPA (QRPA) for superfluid nuclei. Due to the large configuration space, accurate shell model calculations are not feasible for heavy nuclei away from magic numbers $[3,13,14]$. The QRPA approach can be applied to all nuclei in principle except for a few very light systems. While phenomenological QRPA has been quite popular in the past, the self-consistent QRPA approach based on Skyrme [15-21] or relativistic [7,22,23] density functionals has become increasingly accurate and successful in reproducing the observed properties of chargeexchange excitations.

At the RPA level, the nuclear collective motion is treated as a superposition of one-particle-one-hole (1p-1h) excitations; in the QRPA case, this becomes a superposition of twoquasiparticle (2qp) excitations. However, the energy and angular momentum of the collective motion can be transferred to more complicated nuclear states having $2 \mathrm{p}-2 \mathrm{~h}, \ldots, n \mathrm{p}-n \mathrm{~h}$ character (or 4qp, .., nqp character in the superfluid case). This produces the spreading width of giant resonances. In general, the (Q)RPA approach is not able to describe the fragmentation and the detailed line shape of the multipole response. The RPA plus particle-vibration coupling (RPA+PVC) is 
an extension of the RPA approach which has turned out to be quite effective, and in which the $1 \mathrm{p}-1 \mathrm{~h}$ configurations are coupled to collective vibrational phonons [24-26]. The self-consistent RPA+PVC approach for the charge-exchange excitations has been established within both the relativistic $[27,28]$ and the nonrelativistic framework [29,30]. In both cases, it has been possible to show that a conspicuous spreading width is developed with the inclusion of PVC effects, and thus good agreement with experimental data for the GTR and the SDR is obtained. The RPA+PVC model has also been applied to $\beta$ decay [31], and great improvement with respect to mere RPA has been found as far as the description of the $\beta$-decay half-lives in magic nuclei is concerned.

The RPA+PVC approach is obviously limited to the case of magic nuclei. In this paper we extend the formalism to the case of spherical superfluid nuclei, describing the nuclear ground state within the Hartree-Fock-Bogoliubov (HFB) approximation, and the collective excitations of the system within QRPA. Although we will only consider the well bound nucleus ${ }^{120} \mathrm{Sn}$ in this study, we notice that our consistent treatment of mean-field and pairing correlations will be crucial for future studies of exotic nuclei far from the valley of stability. In fact, in these systems the GT strength is expected to move from the giant resonance region to lower energies, where the transitions involving weakly bound and continuum nucleon states play a relevant role.

We focus here mainly on the main features of the QRPA+QPVC model. As we would like to discuss in detail the physical effects inherent in QRPA+QPVC, we shall consider the nucleus ${ }^{120} \mathrm{Sn}$. This is a paradigmatic superfluid nucleus that has been taken as a benchmark in many calculations. In particular, it has been shown that the coupling between quasiparticle and vibrational degrees of freedom explains the lowlying spectra of this and of the neighboring nuclei in a quite convincing way (see, e.g., [32,33] and references therein).

Another point is that in superfluid nuclei both isovector (IV) and isoscalar (IS) pairing are expected to play a relevant role. While the usual IV pairing determines the ground-state structure, the IS pairing is present in the QRPA residual interaction for Gamow-Teller transitions. In our previous works $[18,34,35]$, we have shown the importance of the GT data to pin down the value of the IS pairing strength. Consequently, the role of such IS pairing in calculations beyond QRPA should also be assessed.

A similar model has been recently proposed within the relativistic framework, and applied to different giant resonances (cf., e.g., Ref. [36] where results for the giant dipole resonance (GDR) in ${ }^{120} \mathrm{Sn}$ have been presented). This model has also been applied for the study of the GT response and the $\beta$-decay half-lives in Ni isotopes [37]. While the relativistic model is similar in spirit to the present one, we stress again that our goal is to address in detail the microscopic mechanisms related to quasiparticle-vibration coupling and, to some extent, also to IS pairing.

The paper is organized as follows. In Sec. II and III, the formulas and numerical details of the QRPA+QPVC model are presented. In Sec. IV, the GT response of ${ }^{120} \mathrm{Sn}$ is illustrated and a detailed analysis is provided. Finally, the main conclusions of this work are summarized in Sec. V.

\section{FORMALISM}

We first carry out a self-consistent HFB+QRPA calculation of the GT strength, using a standard Skyrme interaction. The detailed formulas of charge-exchange QRPA on top of HFB can be found in Ref. [18]. It should be noticed that besides the isovector $T=1$ pairing both in the ground state and in the residual interaction, the isoscalar $T=0$ pairing must also be included in the residual interaction in the QRPA calculation. The necessity of isoscalar $T=0$ pairing has been discussed in many previous works, especially in connection with the lowlying GT strength of $N=Z+2$ nuclei and the $\beta$-decay halflives [7,18,34,38-41]. We adopt a density-dependent, zerorange surface pairing force parametrized as follows [18]:

$$
\begin{aligned}
& V_{T=1}\left(\mathbf{r}_{1}, \mathbf{r}_{2}\right)=V_{0} \frac{1-P_{\sigma}}{2}\left(1-\frac{\rho(\mathbf{r})}{\rho_{0}}\right) \delta\left(\mathbf{r}_{1}-\mathbf{r}_{2}\right), \\
& V_{T=0}\left(\mathbf{r}_{1}, \mathbf{r}_{2}\right)=f V_{0} \frac{1+P_{\sigma}}{2}\left(1-\frac{\rho(\mathbf{r})}{\rho_{0}}\right) \delta\left(\mathbf{r}_{1}-\mathbf{r}_{2}\right),
\end{aligned}
$$

where $\mathbf{r}=\left(\mathbf{r}_{1}+\mathbf{r}_{2}\right) / 2, \rho_{0}$ is taken to be $\rho_{0}=0.16 \mathrm{fm}^{-3}$, and $P_{\sigma}$ is the spin exchange operator. Although the $T=0$ pairing strength has not yet been very firmly constrained, several different types of analysis are consistent with values of the proportionality factor $f$ which are close to 1 , or slightly larger [35]. Accordingly, in this work we adopt the two values $f=0$ and $f=1$. This allows the reader to pin down the effect of $T=0$ pairing, by comparing results with a typically accepted strength with results in which it has been completely neglected.

The GT excitations are obtained by the diagonalization of the QRPA matrix. Forward-going and backward-going amplitudes associated with the QRPA eigenstates $|n\rangle$ will be denoted by $X_{a b}^{(n)}$ and $Y_{a b}^{(n)}$, respectively. Here and in what follows, the indices $a, b$, etc., label the so-called BCS quasiparticle states in the canonical bases, that are those defined by the operators $\alpha$ and $\alpha^{\dagger}$ at p. 248 of Ref. [42]. Within QPVC, the QRPA strength will be shifted and redistributed through the coupling to a set of doorway states, denoted by $|N\rangle$, made of a two BCS quasiparticle excitation $|a b\rangle$ coupled to a collective vibration $|n L\rangle$ of angular momentum $L$ and energy $\omega_{n L}$. The properties of these collective vibrations, i.e., phonons $|n L\rangle$ are, in turn, obtained by computing the QRPA response with the same Skyrme interaction, for states of natural parity $L^{\pi}=0^{+}, 1^{-}, 2^{+}, 3^{-}, 4^{+}, 5^{-}$, and $6^{+}$. We have retained the phonons with energy less than $20 \mathrm{MeV}$ and that absorb a fraction of the non-energy-weighted isoscalar or isovector sum rule (NEWSR) strength larger than 5\%. In the case of the Skyrme sets used in this work, the total number of phonons included in the calculation is 25 for the interactions SkM* [43] and SGII [44], and 22 for the interaction SAMi [45].

The GT strength associated with QRPA+QPVC is given by

$$
S(E)=-\frac{1}{\pi} \operatorname{Im} \sum_{\nu}\left\langle 0\left|\hat{O}_{\mathrm{GT}^{ \pm}}\right| v\right\rangle^{2} \frac{1}{E-\Omega_{v}+i\left(\frac{\Gamma_{v}}{2}+\Delta\right)},
$$

where the GT operator is $\hat{O}_{\mathrm{GT}^{ \pm}}=\sum_{i=1}^{A} \sigma(i) t_{ \pm}(i)$ and $\Delta$ is a smearing parameter. In our calculation, we will only focus on the $\mathrm{GT}^{-}$excitations. $|\nu\rangle$ denote the eigenstates [associated with the complex eigenvalues $\Omega_{v}-i \frac{\Gamma_{v}}{2}$ and eigenvectors 
$\left.\left(F^{(v)}, \bar{F}^{(v)}\right)\right]$ that are obtained by diagonalizing the energydependent complex matrix

$$
\begin{aligned}
& \left(\begin{array}{cc}
\mathcal{D}+\mathcal{A}_{1}(E) & \mathcal{A}_{2}(E) \\
-\mathcal{A}_{3}(E) & -\mathcal{D}-\mathcal{A}_{4}(E)
\end{array}\right)\left(\begin{array}{l}
F^{(v)} \\
\bar{F}^{(v)}
\end{array}\right) \\
& =\left(\Omega_{v}-i \frac{\Gamma_{v}}{2}\right)\left(\begin{array}{l}
F^{(v)} \\
\bar{F}^{(v)}
\end{array}\right) .
\end{aligned}
$$

$\mathcal{D}$ is a diagonal matrix containing the physical QRPA eigenvalues. The $\mathcal{A}_{i}$ matrices are complex and energy dependent, associated with the coupling to the doorway states. The expressions of $\mathcal{A}_{i}$ in the QRPA basis $|n\rangle$ are given by

$$
\begin{aligned}
\left(\mathcal{A}_{1}\right)_{m n}= & \sum_{a b, a^{\prime} b^{\prime}} W_{a b, a^{\prime} b^{\prime}}^{\downarrow}(E) X_{a b}^{(m)} X_{a^{\prime} b^{\prime}}^{(n)} \\
& +W_{a b, a^{\prime} b^{\prime}}^{\downarrow *}(-E) Y_{a b}^{(m)} Y_{a^{\prime} b^{\prime}}^{(n)}, \\
\left(\mathcal{A}_{2}\right)_{m n}= & \sum_{a b, a^{\prime} b^{\prime}} W_{a b, a^{\prime} b^{\prime}}^{\downarrow}(E) X_{a b}^{(m)} Y_{a^{\prime} b^{\prime}}^{(n)} \\
& +W_{a b, a^{\prime} b^{\prime}}^{\downarrow *}(-E) Y_{a b}^{(m)} X_{a^{\prime} b^{\prime}}^{(n)}, \\
\left(\mathcal{A}_{3}\right)_{m n}= & \sum_{a b, a^{\prime} b^{\prime}} W_{a b, a^{\prime} b^{\prime}}^{\downarrow}(E) Y_{a b}^{(m)} X_{a^{\prime} b^{\prime}}^{(n)} \\
& +W_{a b, a^{\prime} b^{\prime}}^{\downarrow *}(-E) X_{a b}^{(m)} Y_{a^{\prime} b^{\prime}}^{(n)}, \\
\left(\mathcal{A}_{4}\right)_{m n}= & \sum_{a b, a^{\prime} b^{\prime}} W_{a b, a^{\prime} b^{\prime}}^{\downarrow}(E) Y_{a b}^{(m)} Y_{a^{\prime} b^{\prime}}^{(n)} \\
& +W_{a b, a^{\prime} b^{\prime}}^{\downarrow *}(-E) X_{a b}^{(m)} X_{a^{\prime} b^{\prime}}^{(n)} .
\end{aligned}
$$

To speed up the calculation, we will include in the calculation only states (in both $T_{-}$and $T_{+}$channels) associated with a transition strength larger than a given threshold. Note that the $T_{-}$and $T_{+}$channels are coupled in the QRPA and QRPA+QPVC matrices, when both protons and neutrons are superfluid, at variance with the case of RPA and RPA+PVC (and with the case in which only one of the two species is superfluid, as in $\left.{ }^{120} \mathrm{Sn}\right)$. The matrix $\left(\begin{array}{cc}\mathcal{D}+\mathcal{A}_{1}(E) & \mathcal{A}_{2}(E) \\ \mathcal{A}_{3}(E) & \mathcal{D}+\mathcal{A}_{4}(E)\end{array}\right)$ is still symmetric as in the RPA+PVC case.

The spreading matrix $W_{a b, a^{\prime} b^{\prime}}^{\downarrow}(E)$ is the most important quantity in the QRPA+QPVC model, and it has a more general form than that in the RPA+PVC case:

$$
\begin{aligned}
W_{a b, a^{\prime} b^{\prime}}^{\downarrow} & =\left\langle a b\left|V \frac{1}{E-\hat{H}} V\right| a^{\prime} b^{\prime}\right\rangle \\
& =\sum_{N N^{\prime}}\langle a b|V| N\rangle\left\langle N\left|\frac{1}{E-\hat{H}}\right| N^{\prime}\right\rangle\left\langle N^{\prime}|V| a^{\prime} b^{\prime}\right\rangle,
\end{aligned}
$$

where $|N\rangle=\left|a^{\prime \prime} b^{\prime \prime}\right\rangle \otimes|n L\rangle$ represents a doorway state and $a^{\prime \prime}, b^{\prime \prime}$ are BCS quasiparticle states, as recalled above. $|n L\rangle$ is the $n$th phonon state with the multipolarity $L$. The first term of Eq. (9) is

$$
\langle a b|V| N\rangle=\left\langle a b|V| a^{\prime \prime} b^{\prime \prime} \otimes n L\right\rangle=\left\langle 0\left|\alpha_{b} \alpha_{a} V \alpha_{a^{\prime \prime}}^{\dagger} \alpha_{b^{\prime \prime}}^{\dagger} \Gamma_{n L}^{\dagger}\right| 0\right\rangle,
$$

where $\alpha_{a}$ and $\alpha_{a}^{\dagger}$ are the annihilation and creation operator for the BCS quasiparticle with quantum numbers $a \equiv\{n l j\}$, and $\Gamma_{n L}^{\dagger}$ is the creation operator for phonons. The operator $\Gamma_{n L}^{\dagger}$ has the following form

$$
\Gamma_{n L}^{\dagger}=\frac{1}{\sqrt{1+\delta_{c d}}} \sum_{c \geqslant d} X_{c d}^{(n)} \alpha_{c}^{\dagger} \alpha_{d}^{\dagger}-Y_{c d}^{(n)} \alpha_{d} \alpha_{c},
$$

where $X_{c d}^{(n)}$ and $Y_{c d}^{(n)}$ are the phonon forward and backward QRPA amplitudes. Finally, we arrive at

$$
\langle a b|V| N\rangle=\delta_{b b^{\prime \prime}}\left\langle a|V| a^{\prime \prime}, n L\right\rangle+\delta_{a a^{\prime \prime}}\left\langle b|V| b^{\prime \prime}, n L\right\rangle,
$$

where

$$
\begin{aligned}
\left\langle a^{\prime \prime}, n L|V| a\right\rangle= & \left\langle a|V| a^{\prime \prime}, n L\right\rangle \\
= & \frac{1}{\sqrt{1+\delta_{c d}}} \sum_{c \geqslant d}\left\{\left[V_{a \bar{d} a^{\prime \prime} c}\left(u_{a^{\prime \prime}} u_{c} v_{d} u_{a}-v_{a^{\prime \prime}} v_{c} u_{d} v_{a}\right)+V_{a \bar{a}^{\prime \prime} c d}\left(u_{c} u_{d} v_{a^{\prime \prime}} u_{a}-v_{c} v_{d} u_{a^{\prime \prime}} v_{a}\right)\right.\right. \\
& \left.+V_{a \bar{c} d a^{\prime \prime}}\left(u_{d} u_{a^{\prime \prime}} v_{c} u_{a}-v_{d} v_{a^{\prime \prime}} u_{c} v_{a}\right)\right] X_{c d} \\
& +\left[V_{a^{\prime \prime}} \bar{d} a c\right. \\
& \left.\left.+V_{a^{\prime \prime}} \bar{c} d a u_{a}\left(u_{d} u_{a} v_{c} v_{c} u_{a^{\prime \prime}}-v_{d} v_{c} u_{d} u_{c} v_{a^{\prime \prime}}\right)\right] Y_{c d}\right\} .
\end{aligned}
$$

The above matrix elements $V$ are calculated in the canonical basis. $v_{a}$ is the square root of the occupation probability for the state $a$ in the canonical basis, and $u_{a}=\sqrt{1-v_{a}^{2}}$ is the unoccupied amplitude. The detailed derivation of Eqs. (12) and (13) can be found in Appendix A.

In the second term of Eq. (9), we have

$$
\left\langle N\left|\frac{1}{E-\hat{H}}\right| N^{\prime}\right\rangle=\left\langle a^{\prime \prime} b^{\prime \prime} \otimes n L\left|\frac{1}{E-\hat{H}}\right| a^{\prime \prime \prime} b^{\prime \prime \prime} \otimes n^{\prime} L^{\prime}\right\rangle .
$$

We will express this formula in terms of HFB quasiparticle states $|\tilde{a} \tilde{b}\rangle$ of energy $E_{\tilde{a}}+E_{\tilde{b}}$ and we will assume that the configurations $|\tilde{N}\rangle=|\tilde{a} \tilde{b} \otimes n L\rangle$ do not interact and are eigenstates of the Hamiltonian $\hat{H}$ with eigenvalues $E_{\tilde{a}}+E_{\tilde{b}}+$ $\omega_{n L}$. We then obtain

$$
\begin{aligned}
& \left\langle N\left|\frac{1}{E-\hat{H}}\right| N^{\prime}\right\rangle \\
& \quad=\sum_{\tilde{a}^{\prime \prime} \tilde{b}^{\prime \prime}} \frac{C_{a^{\prime \prime} \tilde{a}^{\prime \prime}}}{E-\left[E_{\tilde{a}^{\prime \prime}}+E_{\tilde{b}^{\prime \prime}}+\omega_{n L} \pm\left(\lambda_{n}-\lambda_{p}\right)\right]+i \Delta},
\end{aligned}
$$

where $C$ represents the unitary transformation matrix between HFB quasiparticle states and BCS quasiparticle states, as 
defined at p. 248 of Ref. [42]. The chemical potential difference $\lambda_{n}-\lambda_{p}$ is included in the energy denominator so that it can reproduce the RPA+PVC limit for magic nuclei, where the sign "-" is for $T_{-}$excitations and and "+" for $T_{+}$excitations. The smearing parameter $\Delta$ is introduced to avoid singularities in the denominator, and a convenient practical value is $\Delta=$ $200 \mathrm{keV}$. Such a value is usually smaller than $\Gamma_{v} / 2$ and does not affect appreciably the QRPA+QPVC calculation of the strength in Eq. (3).

With the above expressions, we calculate the $W_{a b, a^{\prime} b^{\prime}}^{\downarrow}$ matrix elements, and obtain them as the sum of four terms. In the spherical case, we can write all the formulas in angular momentum coupled form. The detailed derivation can be found in the Appendix B. The final expression for $W_{a b, a^{\prime} b^{\prime}}^{\downarrow J}$ reads

$$
\begin{aligned}
& W_{1 a b, a^{\prime} b^{\prime}}^{\downarrow J}=\delta_{j_{b} j_{b^{\prime}}} \delta_{l_{b} l_{b^{\prime}}} \delta_{j_{a} j_{a^{\prime}}} \frac{1}{\hat{j}_{a}^{2}} \sum_{a^{\prime \prime} a^{\prime \prime} \tilde{a}^{\prime \prime} \tilde{b}^{\prime \prime}} \sum_{n L} \delta_{j_{a^{\prime \prime}} j_{a^{\prime \prime \prime}}} \delta_{l_{a^{\prime \prime}} l_{a^{\prime \prime \prime}}} \frac{\left\langle a\|V\| a^{\prime \prime}, n L\right\rangle C_{a^{\prime \prime} \tilde{a}^{\prime \prime}} C_{b \tilde{b}^{\prime \prime}} C_{\tilde{a}^{\prime \prime} a^{\prime \prime \prime}}^{\dagger} C_{\tilde{b}^{\prime \prime} b^{\prime}}^{\dagger}\left\langle a^{\prime}\|V\| a^{\prime \prime \prime}, n L\right\rangle}{E-\left[E_{\tilde{a}^{\prime \prime}}+E_{\tilde{b}^{\prime \prime}}+\omega_{n L} \pm\left(\lambda_{n}-\lambda_{p}\right)\right]+i \Delta}, \\
& W_{2 a b, a^{\prime} b^{\prime}}^{\downarrow J}=\delta_{j_{a} j_{a^{\prime}}} \delta_{l_{a} l_{a^{\prime}}} \delta_{j_{b} j_{b^{\prime}}} \frac{1}{\hat{j}_{b}^{2}} \sum_{b^{\prime \prime} b^{\prime \prime \prime} \tilde{a}^{\prime \prime} \tilde{b}^{\prime \prime}} \sum_{n L} \delta_{j_{b^{\prime \prime}} j_{b^{\prime \prime \prime}}} \delta_{l_{b^{\prime \prime}} l_{b^{\prime \prime \prime}}} \frac{\left\langle b\|V\| b^{\prime \prime}, n L\right\rangle C_{a \tilde{a}^{\prime \prime}} C_{b^{\prime \prime} \tilde{b}^{\prime \prime}} C_{\tilde{a}^{\prime \prime} a^{\prime}}^{\dagger} C_{\tilde{b}^{\prime \prime} b^{\prime \prime \prime}}^{\dagger}\left\langle b^{\prime}\|V\| b^{\prime \prime \prime}, n L\right\rangle}{E-\left[E_{\tilde{a}^{\prime \prime}}+E_{\tilde{b}^{\prime \prime}}+\omega_{n L} \pm\left(\lambda_{n}-\lambda_{p}\right)\right]+i \Delta}, \\
& W_{3 a b, a^{\prime} b^{\prime}}^{\downarrow J}=(-)^{j_{a}+j_{b}+J}\left\{\begin{array}{lll}
j_{a} & j_{b} & J \\
j_{b^{\prime}} & j_{a^{\prime}} & L
\end{array}\right\} \sum_{a^{\prime \prime \prime} b^{\prime \prime} \tilde{a}^{\prime \prime} \tilde{b}^{\prime \prime}} \sum_{n L} \delta_{j_{b^{\prime \prime}} j_{b^{\prime}}} \delta_{l_{b^{\prime \prime}}} l_{b^{\prime}} \delta_{j_{a^{\prime \prime \prime}} j_{a}} \delta_{l_{a^{\prime \prime \prime}} l_{a}} \frac{\left\langle b\|V\| b^{\prime \prime}, n L\right\rangle C_{a \tilde{a}^{\prime \prime}} C_{b^{\prime \prime} \tilde{b}^{\prime \prime}} C_{\tilde{a}^{\prime \prime} a^{\prime \prime \prime}}^{\dagger} C_{\tilde{b}^{\prime \prime} b^{\prime}}^{\dagger}\left\langle a^{\prime}\|V\| a^{\prime \prime \prime}, n L\right\rangle}{E-\left[E_{\tilde{a}^{\prime \prime}}+E_{\tilde{b}^{\prime \prime}}+\omega_{n L} \pm\left(\lambda_{n}-\lambda \lambda_{p}\right)\right]+i \Delta}, \\
& W_{4 a b, a^{\prime} b^{\prime}}^{\downarrow J}=(-)^{j_{a^{\prime}}+j_{b^{\prime}}+J}\left\{\begin{array}{ccc}
j_{a^{\prime}} & j_{b^{\prime}} & J \\
j b & j_{a} & L
\end{array}\right\} \sum_{b^{\prime \prime \prime} a^{\prime \prime} \tilde{a}^{\prime \prime} \tilde{b}^{\prime \prime}} \sum_{n L} \delta_{j_{a^{\prime \prime}} j_{a^{\prime}}} \delta_{l_{a^{\prime \prime}} l_{a^{\prime}}} \delta_{j_{b^{\prime \prime}} j_{b}} \delta_{l_{b^{\prime \prime \prime}} l_{b}} \frac{\left\langle a\|V\| a^{\prime \prime}, n L\right\rangle C_{b \tilde{b}^{\prime \prime}} C_{a^{\prime \prime} \tilde{a}^{\prime \prime}} C_{\tilde{b}^{\prime \prime} b^{\prime \prime \prime}}^{\dagger} C_{\tilde{a}^{\prime \prime} a^{\prime}}^{\dagger}\left\langle b^{\prime}\|V\| b^{\prime \prime \prime}, n L\right\rangle}{E-\left[E_{\tilde{a}^{\prime \prime}}+E_{\tilde{b}^{\prime \prime}}+\omega_{n L} \pm\left(\lambda_{n}-\lambda_{p}\right)\right]+i \Delta}
\end{aligned}
$$

In the above formulas, $\hat{j}_{i}^{2}$ is a shorthand notation for $2 j_{i}+1$. The reduced matrix element has the following form:

$$
\left\langle a\|V\| a^{\prime \prime}, n L\right\rangle=\frac{\hat{L}}{\sqrt{1+\delta_{c d}}} \sum_{c d}\left[\widetilde{V}\left(c d L a^{\prime \prime} ; a\right) X_{c d}^{n L}+(-1)^{j_{a}-j_{a^{\prime \prime}}+L} \widetilde{V}\left(c d L a ; a^{\prime \prime}\right) Y_{c d}^{n L}\right]
$$

where

$\widetilde{V}\left(c d L a^{\prime \prime} ; a\right)=V_{a d a^{\prime \prime} c}^{L p h}\left(u_{a} u_{a^{\prime \prime}} u_{c} v_{d}-v_{a} v_{a^{\prime \prime}} v_{c} u_{d}\right)+V_{a c a^{\prime \prime} d}^{L p h}\left(u_{a} u_{a^{\prime \prime}} v_{c} u_{d}-v_{a} v_{a^{\prime \prime}} u_{c} v_{d}\right)(-)^{j_{c}-j_{d}+L}-V_{a a^{\prime \prime} c d}^{L p p}\left(u_{a} v_{a^{\prime \prime}} u_{c} u_{d}-v_{a} u_{a^{\prime \prime}} v_{c} v_{d}\right)$

and

$\tilde{V}\left(c d L a ; a^{\prime \prime}\right)=V_{a^{\prime \prime} d a c}^{L p h}\left(u_{a} u_{a^{\prime \prime}} u_{c} v_{d}-v_{a} v_{a^{\prime \prime}} v_{c} u_{d}\right)+V_{a^{\prime \prime} c a d}^{L p h}\left(u_{a} u_{a^{\prime \prime}} v_{c} u_{d}-v_{a} v_{a^{\prime \prime}} u_{c} v_{d}\right)(-)^{j_{c}-j_{d}+L}-V_{a^{\prime \prime} a c d}^{L p p}\left(v_{a} u_{a^{\prime \prime}} u_{c} u_{d}-u_{a} v_{a^{\prime \prime}} v_{c} v_{d}\right)$.

This expression for $\left\langle a\|V\| a^{\prime \prime}, n L\right\rangle$ turns out to be in agreement with Ref. [46]. The ph and pp interaction will take the same form as that used for non-charge-exchange QRPA calculation. The ph and pp matrix elements $V_{a b c d}^{L p h}$ and $V_{a b c d}^{L p p}$ are in their angular momentum coupled form,

$$
\begin{aligned}
V_{a b c d}^{L p h} & =\sum_{m_{a} m_{b} m_{c} m_{d}}\left\langle j_{a} m_{a} j_{c}-m_{c} \mid L M\right\rangle(-)^{j_{c}-m_{c}}\left\langle j_{d} m_{d} j_{b}-m_{b} \mid L M\right\rangle(-)^{j_{b}-m_{b}} V_{a b c d}^{p h}, \\
V_{a b c d}^{L p p} & =\sum_{m_{a} m_{b} m_{c} m_{d}}\left\langle j_{a} m_{a} j_{b} m_{b} \mid L M\right\rangle\left\langle j_{c} m_{c} j_{d} m_{d} \mid L M\right\rangle V_{a b c d}^{p p} .
\end{aligned}
$$

For nuclei not far from the stability line, like the nucleus ${ }^{120} \mathrm{Sn}$ studied in this work, the BCS quasiparticle states represent a convenient and accurate approximation to the HFB states. The corresponding expression for the spreading matrix elements is 
obtained by approximating the $C$ transformation with the identity, that is, putting $C_{a \tilde{a}^{\prime \prime}}=\delta_{a \tilde{a}^{\prime \prime}}$ in Eqs. (16)-(19). One then obtains

$$
\begin{aligned}
& W_{1 a b, a^{\prime} b^{\prime}}^{\downarrow J}=\delta_{b b^{\prime}} \delta_{j_{a} j_{a^{\prime}}} \frac{1}{\hat{j}_{a}^{2}} \sum_{a^{\prime \prime}, n L} \frac{\left\langle a\|V\| a^{\prime \prime}, n L\right\rangle\left\langle a^{\prime}\|V\| a^{\prime \prime}, n L\right\rangle}{E-\left[\omega_{n L}+E_{a^{\prime \prime}}+E_{b} \pm\left(\lambda_{n}-\lambda_{p}\right)\right]+i \Delta}, \\
& W_{2 a b, a^{\prime} b^{\prime}}^{\downarrow J}=\delta_{a a^{\prime}} \delta_{j_{b} j_{b^{\prime}}} \frac{1}{\hat{j}_{b}^{2}} \sum_{b^{\prime \prime}, n L} \frac{\left\langle b\|V\| b^{\prime \prime}, n L\right\rangle\left\langle b^{\prime}\|V\| b^{\prime \prime}, n L\right\rangle}{E-\left[\omega_{n L}+E_{b^{\prime \prime}}+E_{a} \pm\left(\lambda_{n}-\lambda_{p}\right)\right]+i \Delta}, \\
& W_{3 a b, a^{\prime} b^{\prime}}^{\downarrow J}=(-)^{j_{a}+j_{b}+J}\left\{\begin{array}{ccc}
j_{a} & j_{b} & J \\
j_{b^{\prime}} & j_{a^{\prime}} & L
\end{array}\right\} \sum_{n L} \frac{\left\langle a^{\prime}\|V\| a, n L\right\rangle\left\langle b\|V\| b^{\prime}, n L\right\rangle}{E-\left[\omega_{n L}+E_{a}+E_{b^{\prime}} \pm\left(\lambda_{n}-\lambda_{p}\right)\right]+i \Delta}, \\
& W_{4 a b, a^{\prime} b^{\prime}}^{\downarrow J}=(-)^{j_{a^{\prime}}+j_{b^{\prime}}+J}\left\{\begin{array}{lll}
j_{a^{\prime}} & j_{b^{\prime}} & J \\
j_{b} & j_{a} & L
\end{array}\right\} \sum_{n L} \frac{\left\langle a\|V\| a^{\prime}, n L\right\rangle\left\langle b^{\prime}\|V\| b, n L\right\rangle}{E-\left[\omega_{n L}+E_{a^{\prime}}+E_{b} \pm\left(\lambda_{n}-\lambda_{p}\right)\right]+i \Delta},
\end{aligned}
$$

where $E_{a}$ is the BCS quasiparticle energy. The four terms correspond to the four diagrams in Fig. 1. These formulas are in agreement with the formulas in Refs. [36] and [37].

\section{NUMERICAL DETAILS}

The HFB code introduced in Ref. [47] is used for the calculation of ground-state properties. The HFB equations are solved in coordinate space on a radial mesh of size $0.1 \mathrm{fm}$, within a spherical box having a radius equal to $20 \mathrm{fm}$. The pairing strength is determined by reproducing the neutron pairing gap in ${ }^{120} \mathrm{Sn}$, which is $\Delta_{n}=1.34 \mathrm{MeV}$.

The configuration space for the QRPA calculation is defined by selecting two quasiparticle states $a$ and $b$ associated with an absolute value of the product $\left|u_{a} v_{b}\right|$ or $\left|u_{b} v_{a}\right|$ (denoted as $|u v|$ ) larger than a given lower cutoff, and with quasiparticle energies smaller than $E_{\text {cut }}$. The same value of $E_{\text {cut }}$ is used for the pairing window in the HFB calculation and the intermediate states of diagrams in Fig. 1 in the QPVC calculation. In order to check the influence of the configuration space, by taking the GT response of ${ }^{120} \mathrm{Sn}$ calculated with the Skyrme interaction SGII as an example, we performed a test of the convergence with respect to the parameters $|u v|$ and $E_{\text {cut }}$ (cf. Fig. 2 and Fig. 3, respectively). From Fig. 2, we can see that when the threshold for the product $|u v|$ is smaller than $10^{-3}$, the GT strength

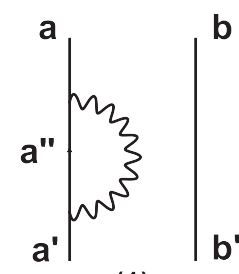

(1)

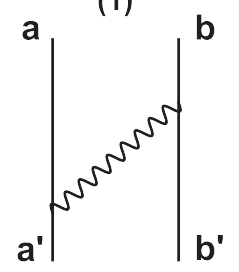

(3)

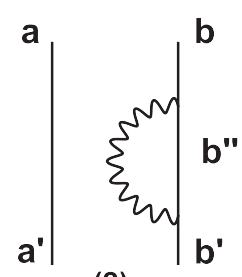

(2)

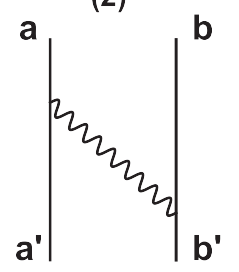

(4)
FIG. 1. Diagrammatic representation of the four terms whose sum gives the matrix element $W_{a b, a^{\prime} b^{\prime}}^{\downarrow}$. distribution is quite stable. Accordingly, the value $|u v|=10^{-3}$ will be adopted as a lower limit in our calculations. In Fig. 3, panels (a) and (b), we check the convergence of the GT strength distribution with respect to $E_{\text {cut }}$ calculated, respectively, within QRPA and QRPA+QPVC. The results are stable for $E_{\text {cut }}$ larger than $60 \mathrm{MeV}$. The value $E_{\text {cut }}=100 \mathrm{MeV}$ will be used in the calculations in Sec. IV. Within the present section, to save computation time we use $E_{\text {cut }}=60 \mathrm{MeV}$.

In the QPVC calculation, Eq. (4) is solved in the QRPA basis. In order to simplify the calculation, we usually neglect QRPA states with very small GT strength, reducing significantly the dimension of the QRPA+QPVC matrix. The influence of the reduction of QRPA basis on the final GT strength distribution is checked in Fig. 4. The cutoff on the relative strength of the QRPA states is denoted as $b_{\text {cut }}$, namely only the QRPA states with a fraction of NEWSR strength larger than $b_{\text {cut }}$ are included in the calculation. We will adopt the value $b_{\text {cut }}=0.001$, which is sufficient for convergence, as shown in Fig. 4.

Finally, we check the sum rule in ${ }^{120} \mathrm{Sn}$, within the QRPA+QPVC calculations, as a function of the number of QRPA basis states obtained by setting $b_{\text {cut }}=10^{-1}, 10^{-2}, 10^{-3}$,

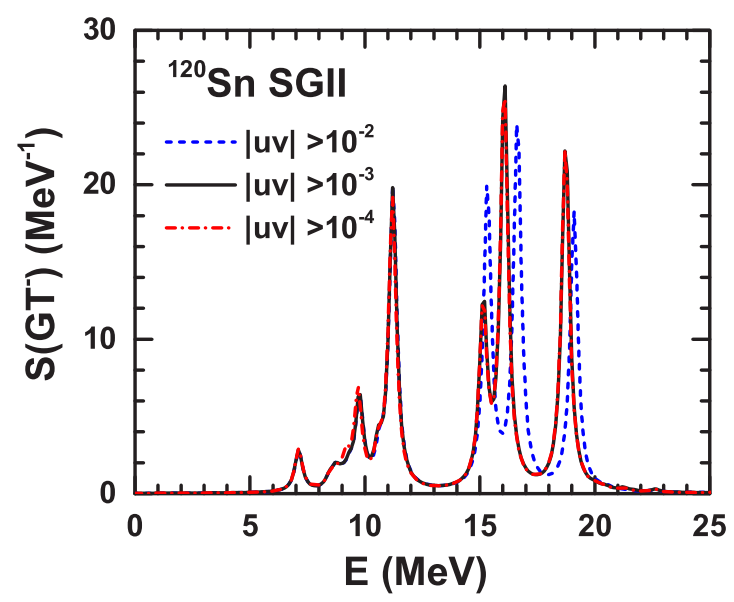

FIG. 2. GT strength function calculated with the interaction SGII within the QRPA approach, using different configuration spaces associated with different threshold values for the product $|u v|$ of the occupation amplitudes in canonical basis. 

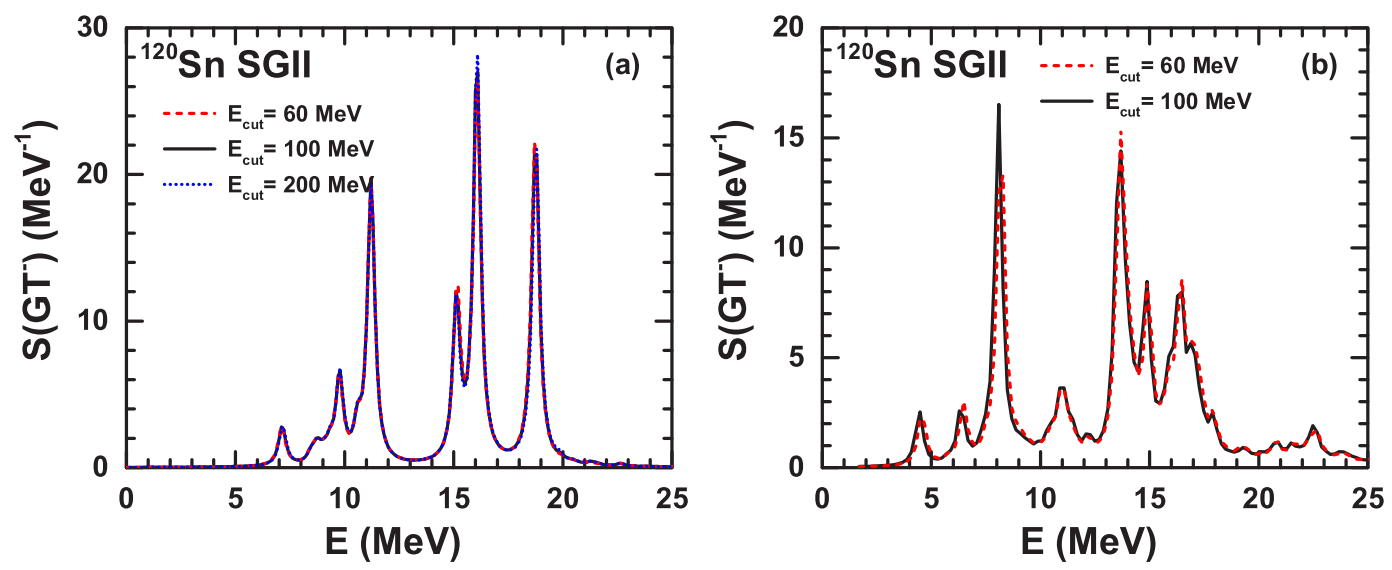

FIG. 3. GT strength function calculated with the interaction SGII within QRPA (a) or QRPA+QPVC (b) with different configuration spaces associated with different values of the energy cutoff $E_{\text {cut }}$.

and $10^{-4}$, in Fig. 5. We consider the integrated strength up to the excitation energy of $80 \mathrm{MeV}$. For $b_{\text {cut }}=10^{-3}$, we obtain $97 \%$ of the Ikeda sum rule.

Previous calculations have been made using the approximation Eq. (25) for the spreading matrix elements. Its validity is checked in Fig. 6 through the comparison between the results with and without the approximation for ${ }^{120}$ Sn using the Skyrme interaction SGII. In order to save computation time, in this case we use $b_{\text {cut }}=0.1$ instead of $b_{\text {cut }}=0.001$. It turns out the two results are in very good agreement with each other. Therefore, in the following QRPA+QPVC calculations, we will use the approximation (25).

In the calculations presented in this section, we have included the isoscalar pairing in QRPA, and we did not adopt the subtraction method in QRPA+QPVC; this topic will be discussed in the next section. The excitation energies are always with respect to the mother nucleus.

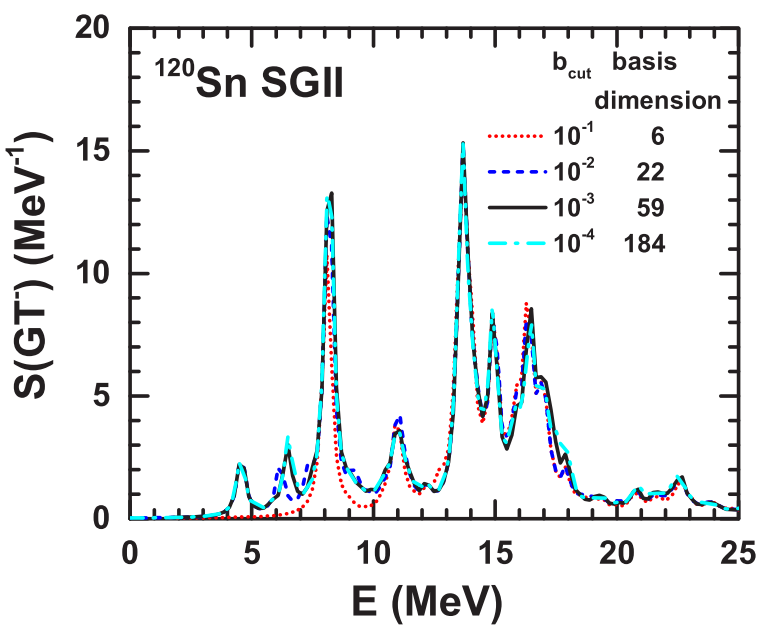

FIG. 4. GT strength function calculated with the interaction SGII within QRPA+QPVC with different configuration spaces associated with different values of the strength cutoff $b_{\text {cut }}$.

\section{RESULTS AND DISCUSSIONS}

Before we proceed with the discussion of our results, we would like to introduce the so-called "subtraction" method. The parameters of the energy density functional (EDF) are optimized to reproduce in the best way nuclear ground state properties, and therefore "static" correlations are implicitly taken into account. When processes beyond mean field are explicitly considered in extended RPA approaches based on this EDF, the parameters of the EDF should be, in principle, readjusted to avoid problems of double counting [48]. This is usually not done, and, as an alternative method to avoid the double counting of static correlations, it has been proposed to subtract the energy-independent part of the self-energy [49]. Recently, it has also been found that this procedure guarantees the validity of the stability condition in extensions of the RPA approach [50]. The theoretical foundation and application of the subtraction method were further discussed within the formalism of second RPA in Ref. [51].

Since we never used the subtraction method in our previous works $[7,9,31]$, we would like to explore the effects of its

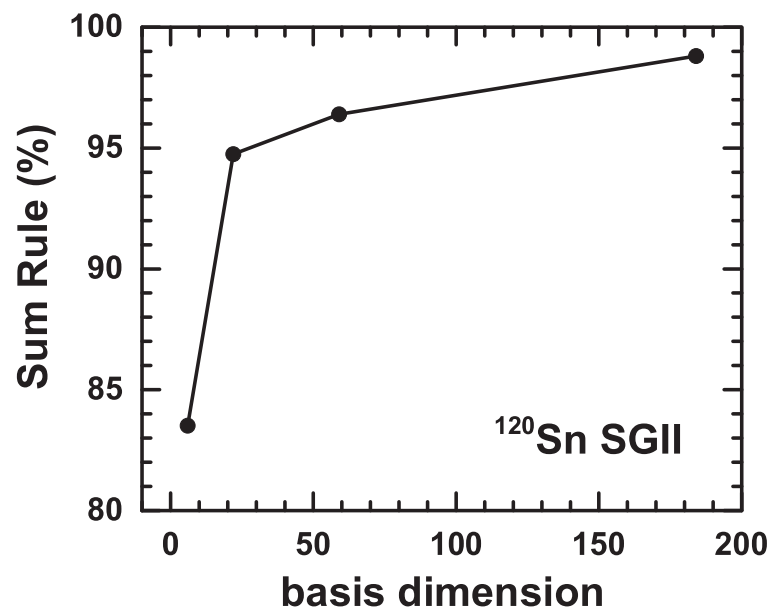

FIG. 5. Ikeda sum rule fulfillment as a function of the number of QRPA basis states used for the QPVC calculation, in the case of the Gamow-Teller response of ${ }^{120} \mathrm{Sn}$ calculated with the interaction SGII. 


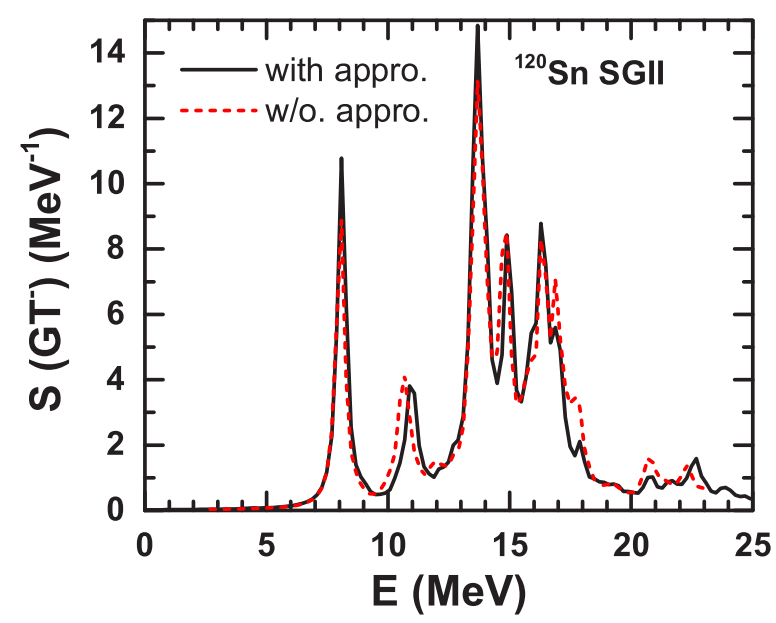

FIG. 6. Gamow-Teller strength distribution in ${ }^{120} \mathrm{Sn}$ calculated by means of the Skyrme QRPA+QPVC model with and without the approximation of Eq. (25) in the spreading matrix elements.

introduction in our present work. We correspondingly modify the QRPA+QPVC equation (4), by writing

$$
\begin{aligned}
& \left(\begin{array}{cc}
\mathcal{D}+\mathcal{A}_{1}(E)-\mathcal{A}_{1}(0) & \mathcal{A}_{2}(E)-\mathcal{A}_{2}(0) \\
-\mathcal{A}_{3}(E)+\mathcal{A}_{3}(0) & -\mathcal{D}-\mathcal{A}_{4}(E)+\mathcal{A}_{4}(0)
\end{array}\right)\left(\begin{array}{l}
F^{(v)} \\
\bar{F}^{(v)}
\end{array}\right) \\
& =\left(\Omega_{v}-i \frac{\Gamma_{v}}{2}\right)\left(\begin{array}{l}
F^{(v)} \\
\bar{F}^{(v)}
\end{array}\right),
\end{aligned}
$$

so that the above equation reduces to the QRPA equation when $E=0$. In practice we just need to introduce the following replacements in Eqs. (5)-(8):

$$
\begin{aligned}
W_{a b a^{\prime} b^{\prime}}^{\downarrow}(E) & \rightarrow W_{a b a^{\prime} b^{\prime}}^{\downarrow}(E)-W_{a b a^{\prime} b^{\prime}}^{\downarrow}(0), \\
W_{a b a^{\prime} b^{\prime}}^{\downarrow}(-E) & \rightarrow W_{a b a^{\prime} b^{\prime}}^{\downarrow}(-E)-W_{a b a^{\prime} b^{\prime}}^{\downarrow}(0) .
\end{aligned}
$$

In Fig. 7, we show the effects of the subtraction method on the GT strength distribution and its cumulative sum in ${ }^{120} \mathrm{Sn}$, using the Skyrme interaction SkM*. Both panel (a) and panel (b) show that by using the subtraction method the value of real part of the self-energy is reduced, especially at low energy; consequently, in an effective way, in introducing the subtraction method one introduces an upward shift of the
TABLE I. The strength $m_{0}$ and the energy weighted sum rule $m_{1}$, integrated up to energy $E=25 \mathrm{MeV}$, as well as the energy centroid $m_{1} / m_{0}$ in the whole energy range $E=0-25 \mathrm{MeV}$, calculated by $\mathrm{QRPA}, \mathrm{QRPA}+\mathrm{QPVC}$, and $\mathrm{QRPA}+\mathrm{QPVC}$ with the subtraction method in ${ }^{120} \mathrm{Sn}$ using the interaction $\mathrm{SkM}^{*}$.

\begin{tabular}{lccc}
\hline \hline & $m_{0}$ & $m_{1}(\mathrm{MeV})$ & $m_{1} / m_{0}(\mathrm{MeV})$ \\
\hline QRPA & 61.3 & 853.4 & 13.9 \\
QRPA+QPVC & 56.8 & 713.0 & 12.6 \\
QRPA+QPVC+subtraction & 56.1 & 772.6 & 13.8 \\
\hline \hline
\end{tabular}

excitation energies. The shift becomes smaller as the energy increases, and is equal to about $1 \mathrm{MeV}$ in the low-energy region and to about $0.5 \mathrm{MeV}$ in the giant resonance region, until it vanishes at $25 \mathrm{MeV}$. The total GT strengths are the same for the QRPA+QPVC calculation with and without subtraction. The width in the giant resonance region is essentially not affected, while the width of the third low-energy peak is increased.

The values of the strength $m_{0}$ and of the energy-weighted sum rule $m_{1}$ up to $E=25 \mathrm{MeV}$, with and without the subtraction method, are reported in Table I. The total strengths $m_{0}$ obtained with and without subtraction method are very close. The centroid energy increases by $1.2 \mathrm{MeV}$ when the subtraction method is introduced, becoming very close to the value calculated in QRPA. This indicates that static correlations are removed by the use of the subtraction method. In the following calculations, we will always use the subtraction method, simply indicated by the label "QRPA+QPVC."

We first report the properties of the collective phonons included in our QPVC calculation. The energy and reduced transition probability of the lowest phonons of different multipolarities in ${ }^{120} \mathrm{Sn}$, calculated by QRPA with the three Skyrme interactions SAMi, SGII, and SkM*, are shown in Table II. All the three interactions tend to overestimate the experimental energies, the best results being obtained with SkM*.

We then show in Fig. 8 the GT strength distributions for ${ }^{120} \mathrm{Sn}$ calculated by the QRPA and QRPA+QPVC models using the three Skyrme interactions, and with a small value of the smearing parameter, $\Delta=0.2 \mathrm{MeV}$. We indicate the

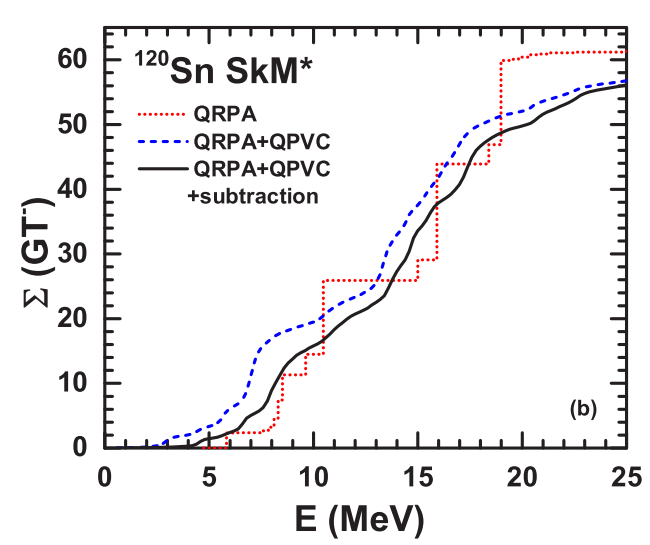

FIG. 7. The Gamow-Teller strength distributions (a) and their cumulative sums (b) for ${ }^{120}$ Sn calculated by means of QRPA and QRPA+QPVC models, without and with subtraction method, using the Skyrme interaction SkM*. 
TABLE II. The energy and reduced transition probability of the lowest phonons of different multipolarities included in the QRPA+QPVC calculation for ${ }^{120} \mathrm{Sn}$. The experimental data are taken from NNDC [52]. The theoretical results are obtained by the QRPA approach with the interactions SAMi, SGII, and SkM*.

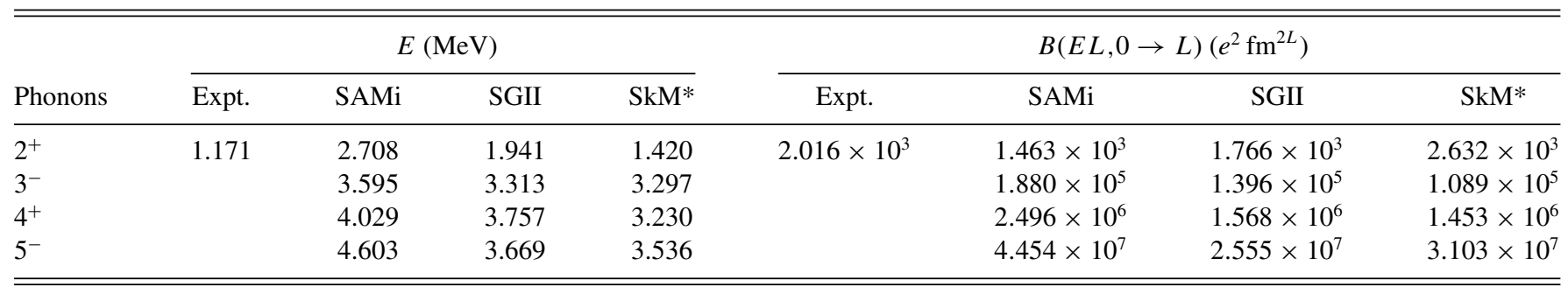

four peak energies identified in the $\left({ }^{3} \mathrm{He}, t\right)$ experiment [53] by dashed lines. The length of each line is proportional to the cross section.

With the interaction SAMi, the peaks obtained in QRPA calculation merge into a single giant resonance peak in the QRPA+QPVC calculation. The peak is narrow, probably due to the too high phonon energies (cf. Table II). The GT strength distribution in the low-energy region is also redistributed and in this case some spreading width is obtained. The QRPA calculation reproduces well the experimental giant resonance peak while the QRPA+QPVC slightly underestimates its energy. As for the interaction SGII, the three QRPA peaks in the giant resonance region merge with the QRPA+QPVC calculation into one resonance peak with some subpeaks, developing a spreading width of about $4.5 \mathrm{MeV}$. We notice that if the subtraction method is not used, the width decreases to $4 \mathrm{MeV}$ (cf. Fig. 4). This is related to the fact that the GTR energies as well as the surface phonon energies are overestimated for this interaction at the QRPA level. The subtraction method then improves the matching between the energy of the GTR energy and of the relevant intermediate configurations in the calculation of the width. Although substantial, the spreading width is still smaller than the experimental value of $6.4 \mathrm{MeV}$ (cf. Figs. 10 and 11 below). The remaining part of the width may be due to the incorrect description of the phonon energies, to some contribution from the escape width, and to correlations coming from the coupling to other states outside our model space. In Fig. 11, this part of width will be simulated by using a larger value of the smearing parameter, $\Delta=0.5 \mathrm{MeV}$. Besides the width, the giant resonance energy is well reproduced in the QRPA+QPVC calculation, while in the low-energy region the agreement with experimental peaks is relatively poor. The SkM* strength distribution in the giant resonance region is quite similar to that obtained with SGII, and displays a spreading width of about $4.8 \mathrm{MeV}$. The overall strength distribution in the low-energy region is better reproduced by $\mathrm{SkM}^{*}$ than by SGII. We will then use only the interaction $\mathrm{SkM}^{*}$ in the rest of our analysis.

In Fig. 9, we plot the Gamow-Teller strength distributions for ${ }^{120} \mathrm{Sn}$ calculated by the QRPA and QRPA+QPVC models, with and without isoscalar pairing. The energies and transition strength of the main GT excitations obtained in QRPA, as well as their main components, are listed in Table III for the case without $(f=0)$ and with $(f=1)$ isoscalar pairing. From Fig. 9(a) as well as Table III, we can see that with the inclusion of the attractive isoscalar pairing the strength in the low-energy region increases, the energies of the peaks at around $10 \mathrm{MeV}$ are shifted downwards, the splitting between the states around $E=15$ and $E=19 \mathrm{MeV}$ becomes smaller, and the strength is redistributed in favor of the lower ones.

Then we analyze in detail the microscopic structures of the main excitations in QRPA. The lowest main state lying at $E=5.96(5.83) \mathrm{MeV}$ for $f=0(f=1)$ is basically a singleparticle excitation of back spin-flip type, i.e., $j=l-1 / 2 \rightarrow$ $j=l+1 / 2$. The second lowest main state at $E=8.62$ (8.51) $\mathrm{MeV}$ for $f=0(f=1)$ is composed of several quasiparticle transitions of non-spin-flip, i.e., $j=l \pm 1 / 2 \rightarrow j=l \pm 1 / 2$ as well as $\Delta L=2$ type. We note that the transitions with $\Delta L=2$ do not contribute to the GT strength, since the GT operator is characterized by $\Delta L=0$. In the $f=0$ case, the
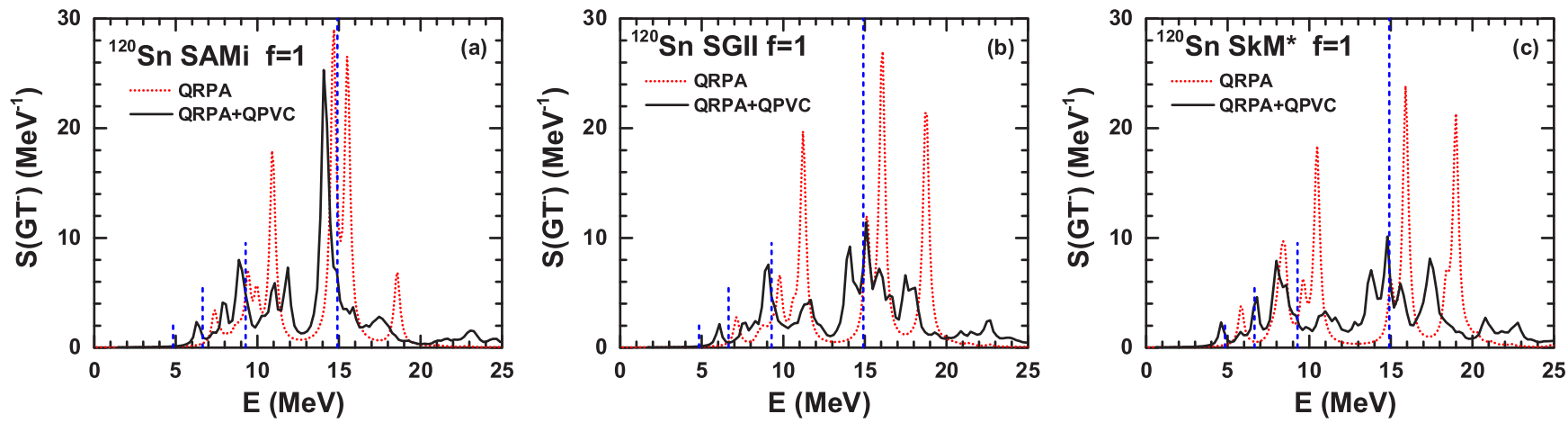

FIG. 8. The Gamow-Teller strength distributions for ${ }^{120} \mathrm{Sn}$ calculated by QRPA and QRPA+QPVC models, with isoscalar pairing, using the interactions SAMi (a), SGII (b), and $\mathrm{SkM}^{*}(\mathrm{c})$. The experimental GT peak energies [53] are denoted by dashed lines, and the length of each line is proportional to the cross section. 


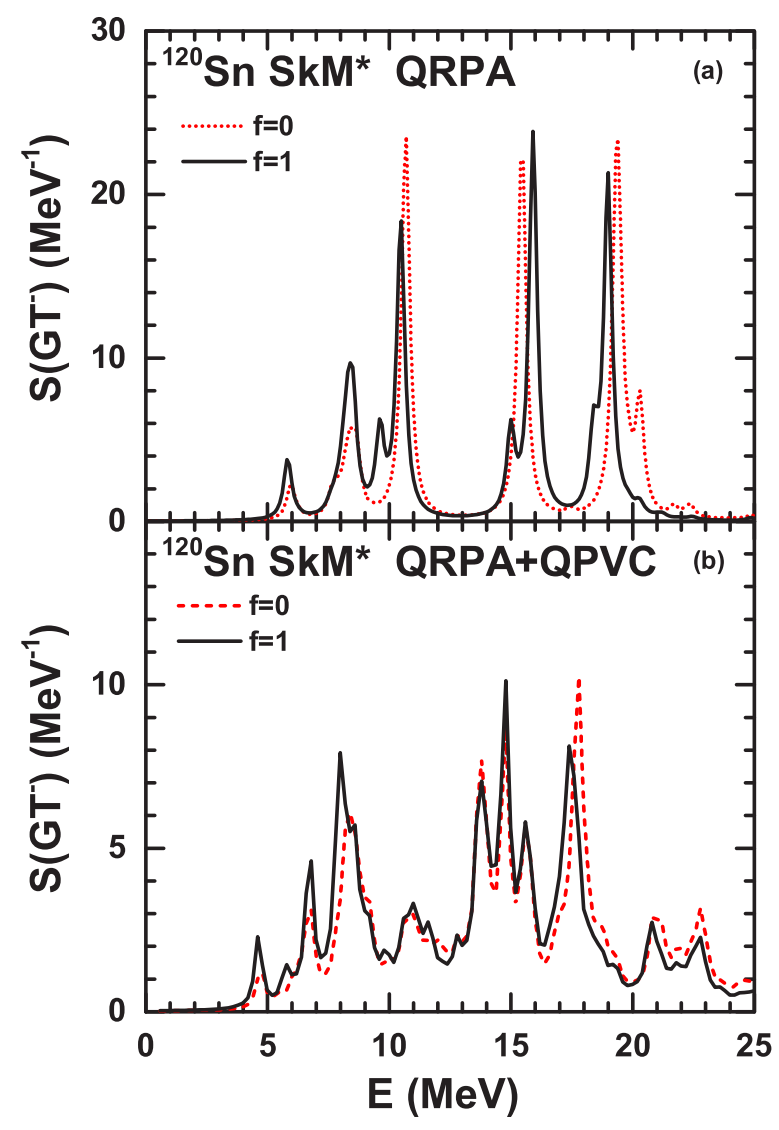

FIG. 9. The Gamow-Teller strength distributions for ${ }^{120} \mathrm{Sn}$ calculated by QRPA (a) and QRPA+QPVC (b) models, with and without isoscalar pairing, using the Skyrme interaction SkM*.

main components of the state at $E=10.67 \mathrm{MeV}$ are a nonspin-flip and a spin-flip transition. When $f=1$, one instead finds two states, one lying at $E=9.62 \mathrm{MeV}$ with a strong non-spin-flip component and another at $10.47 \mathrm{MeV}$ with a strong spin-flip component. In the giant resonance region, in the $f=0$ case, the state at $E=15.45 \mathrm{MeV}$ is composed of the two spin-flip quasi-particle transitions $v 1 g_{9 / 2} \rightarrow \pi 1 g_{7 / 2}$ and $v 1 h_{11 / 2} \rightarrow \pi 1 h_{9 / 2}$. After including the isoscalar pairing, this state splits into two states at $E=15.00$ and $15.91 \mathrm{MeV}$, and other transitions of back spin-flip are mixed into these two states. For $f=0$, the main states in the high energy region lie at $E=19.32,19.43$, and $20.29 \mathrm{MeV}$, and are composed of spin-flip and non-spin-flip quasiparticle transitions. For $f=1$, the highest states lie at $E=18.40$ and $18.98 \mathrm{MeV}$, and the strength is concentrated in the second state. The components of these states are also changed, and the back spin-flip transition $\nu 1 h_{9 / 2} \rightarrow \pi 1 h_{11 / 2}$ plays an important role.

For the QRPA+QPVC results, the profile of the strength function in the giant resonance region is similar in the $f=0$ and $f=1$ cases, although the strengths of the peaks in the low-energy region are increased and the strength of the highest peak is decreased with the inclusion of isoscalar pairing. In the following, we shall discuss the microscopic structure of the GTR peaks for $f=1$.

From the previous section, we recall that at each excitation energy $E$ we solve the QRPA+QPVC equation obtaining a set of eigenstates with complex eigenvalues $\left(\Omega_{v}-i \Gamma_{v} / 2\right)$. We focus on values $E$ corresponding to peaks in the strength function. The contribution to the width is essentially given by twice the imaginary part of the important eigenstates that lie close to $E$. Each of these eigenstates is a linear combination of the QRPA states $|m\rangle$ (with energy $E_{m}$ ). In the following, we will analyze the important eigenstates and their important QRPA components. The eigenstates which give the largest contributions to the strength are given in Table IV, together with the main associated QRPA components $|m\rangle$. For each $|m\rangle$, the most important quasiparticle configurations $a b$ are also listed, together with their contribution to the imaginary part of the self-energy $\mathcal{A}_{1}$. We note that the total width $\Gamma_{v}$ resulting from the complete diagonalization is different from the sum of the values of $\operatorname{Im}\left(\mathcal{A}_{1}\right)_{m m}$, due to the strong mixing between different QRPA states.

The eigenstate with the eigenvalue $(13.65-i 0.34) \mathrm{MeV}$ gives the most important contribution to the peak found at $E=13.79 \mathrm{MeV}$. This eigenstate is mainly composed of the QRPA states at $E=15.00$ and $15.91 \mathrm{MeV}$ (cf. Table III). The contributions to the width from the imaginary parts of the selfenergy of these two QRPA states are -0.57 and $-0.49 \mathrm{MeV}$, respectively. The diagrams $W_{a b a b}$ with $(a, b)=\left(\pi 1 g_{7 / 2}, \nu 1 g_{9 / 2}\right)$ or $\left(\pi 1 h_{9 / 2}, v 1 h_{11 / 2}\right)$ contribute most to the self-energy and, in turn, the coupling to $2^{+}$and $3^{-}$phonons plays the most important role. The same QRPA configurations give the largest contributions to the peak with energy $E=14.79 \mathrm{MeV}$. At the peak energy $E=15.59 \mathrm{MeV}$, the eigenstate with $(15.13-i 1.22) \mathrm{MeV}$ is important, and its main components are again the QRPA states at $E=15.00$ and $15.91 \mathrm{MeV}$. At this peak energy, the imaginary parts of their self-energies are increased to -0.95 and $-0.84 \mathrm{MeV}$, compared to the corresponding values -0.58 and $-0.45 \mathrm{MeV}$ found at the peak energy $E=14.79 \mathrm{MeV}$. The important diagrams are still $W_{a b a b}$ with $(a, b)=\left(\pi 1 g_{7 / 2}, v 1 g_{9 / 2}\right)$ or $\left(\pi 1 h_{9 / 2}, \nu 1 h_{11 / 2}\right)$, but with coupling to $2^{+}, 3^{-}$, and $4^{+}$phonons. At the peak energy $E=17.39 \mathrm{MeV}$, the eigenstates with the eigenvalue (16.89$i 0.68)$ and $(16.90-i 1.14) \mathrm{MeV}$ contribute to the peak. These two states are mainly composed of the QRPA states at $E=15.91,18.40$, and $18.98 \mathrm{MeV}$. The diagram $W_{a b a b}$ with $(a, b)=\left(\pi 1 g_{7 / 2}, \nu 1 g_{9 / 2}\right),\left(\pi 1 h_{9 / 2}, \nu 1 h_{11 / 2}\right),\left(\pi 2 f_{7 / 2}, \nu 2 f_{7 / 2}\right)$, or $\left(\pi 1 h_{9 / 2}, \nu 2 f_{7 / 2}\right)$ contributes to the self-energy most, and the couplings to $2^{+}, 3^{-}, 4^{+}$, and $5^{-}$phonons all play important roles.

In summary, these four subpeaks are mainly composed of several QRPA states in the GTR region. For the first three peaks, the QRPA states at $E=15.00$ and $15.91 \mathrm{MeV}$ are relatively more important, while the QRPA states at $E=15.91,18.40$, and $18.98 \mathrm{MeV}$, as well as the states at even higher energies with small $B(\mathrm{GT})$ values, are important for the fourth peak.

The cumulative sums of the four strength distributions calculated by QRPA and QRPA+QPVC model with and without isoscalar pairing are plotted in Fig. 10. We include for comparison also the experimental results from $\left({ }^{3} \mathrm{He}, t\right)$ and $(p, n)$ reactions. The associated $B(\mathrm{GT})$ strength functions are not given directly in the experimental works of Refs. [53,54]. However, there are some suggestions on how to extract the $B(\mathrm{GT})$ strength functions from the cross sections in these 
TABLE III. QRPA amplitudes $X_{a b}^{2}-Y_{a b}^{2}$ for GT states with large $B(\mathrm{GT})(>1.0)$. The excitation energy $E$ and transition strength $B$ of different GT excitations in ${ }^{120} \mathrm{Sn}$ are calculated in QRPA either without isoscalar pairing $(f=0)$ or with isocalar pairing $(f=1)$. The excitation energies are given with respect to the mother nucleus in units of MeV. For each QRPA state, only the configurations associated with the large QRPA amplitudes $\left(\left|X_{a b}^{2}-Y_{a b}^{2}\right|>0.03\right)$ are listed.

\begin{tabular}{|c|c|c|c|c|c|c|c|c|c|c|c|c|c|c|c|}
\hline \multirow[b]{2}{*}{$E(\mathrm{MeV})$} & \multicolumn{7}{|c|}{$f=0$} & \multicolumn{8}{|c|}{$f=1$} \\
\hline & 5.96 & 8.62 & 10.67 & 15.45 & 19.32 & 19.43 & 20.29 & 5.83 & 8.51 & 9.62 & 10.47 & 15.00 & 15.91 & 18.40 & 18.98 \\
\hline$B(\mathrm{GT})$ & 1.33 & 2.47 & 14.65 & 14.58 & 9.82 & 5.87 & 4.09 & 2.31 & 3.84 & 3.14 & 11.48 & 3.16 & 14.80 & 2.96 & 13.07 \\
\hline$\nu 2 d_{3 / 2} \rightarrow \pi 2 d_{5 / 2}$ & 0.94 & & & & & & & 0.97 & & & & & & & \\
\hline$\nu 2 d_{5 / 2} \rightarrow \pi 2 d_{5 / 2}$ & & 0.04 & 0.04 & & & & & & 0.08 & & & & & & \\
\hline$\nu 2 d_{5 / 2} \rightarrow \pi 1 g_{7 / 2}$ & & 0.15 & & & & & & & 0.11 & 0.29 & & & & & \\
\hline$v 3 s_{1 / 2} \rightarrow \pi 2 d_{3 / 2}$ & & 0.23 & & & & & & & 0.39 & & & & & & \\
\hline$\nu 2 d_{3 / 2} \rightarrow \pi 2 d_{3 / 2}$ & & 0.34 & & & & & & & 0.23 & & & & & & \\
\hline$\nu 1 g_{7 / 2} \rightarrow \pi 1 g_{7 / 2}$ & & 0.11 & & & & & & & 0.09 & & & & & & \\
\hline$v 1 h_{11 / 2} \rightarrow \pi 1 h_{11 / 2}$ & & & 0.28 & & & & & & & 0.61 & & & & & \\
\hline$\nu 2 d_{5 / 2} \rightarrow \pi 2 d_{3 / 2}$ & & & 0.55 & & & & & & & & 0.87 & & & & \\
\hline$v 1 g_{9 / 2} \rightarrow \pi 1 g_{7 / 2}$ & & & & 0.83 & & & 0.06 & & & & & 0.34 & 0.48 & & 0.08 \\
\hline$\nu 3 s_{1 / 2} \rightarrow \pi 5 s_{1 / 2}$ & & & & & & & 0.04 & & & & & & & & \\
\hline$\nu 3 d_{5 / 2} \rightarrow \pi 2 d_{5 / 2}$ & & & & & & & & & & & & & & 0.05 & \\
\hline
\end{tabular}

references. In Ref. [53] that corresponds to the $\left({ }^{3} \mathrm{He}, t\right)$ experiment, the authors provide, in Tables II, III, and IV, the excitation energies, widths and cross sections of four observed GT peaks. Each GT peak can be described by a Gaussian line shape, and the sum of these four Gaussian line shapes gives the total cross section. Furthermore, it is mentioned in Ref. [53] that the integrated $B(\mathrm{GT})$ value of the main component of the GT resonances was found to be $(65 \pm 3) \%$ of the Ikeda sum rule strength of $3(N-Z)$. In Table II of Ref. [53], the total GT cross section is also reported to be $24.2 \pm 2.9 \mathrm{mb} / \mathrm{sr}$. From these values, we obtain the $B(\mathrm{GT})$ strength function by scaling the cross section with a factor of 1.6 to be consistent with the empirical sum rule value $0.65 \times 3(N-Z)=39$. In the case of the $(p, n)$ experiment reported in Ref. [54], not only the cross section $\sigma\left(0^{\circ}\right)$ (in Fig. 4 of [54]), but also the unit cross section $\hat{\sigma}=2.78 \pm 0.16 \mathrm{mb} / \mathrm{sr}$ (in Table I of the same work) were also determined. We can obtain an approximate value for the $B(\mathrm{GT})$ strength, through the relation $\sigma\left(0^{\circ}\right)=\hat{\sigma} F(q, \omega) B(\mathrm{GT})$, by assuming the kinetic correction factor $F(q, \omega)$, which depends on momentum and energy transfer of the cross section, to be constant and equal to 1 . Finally, in order to compare with our theoretical calculations, where the strength functions are calculated with respect to the mother nucleus, both strength functions extracted from $\left({ }^{3} \mathrm{He}, t\right)$ and $(p, n)$ reactions
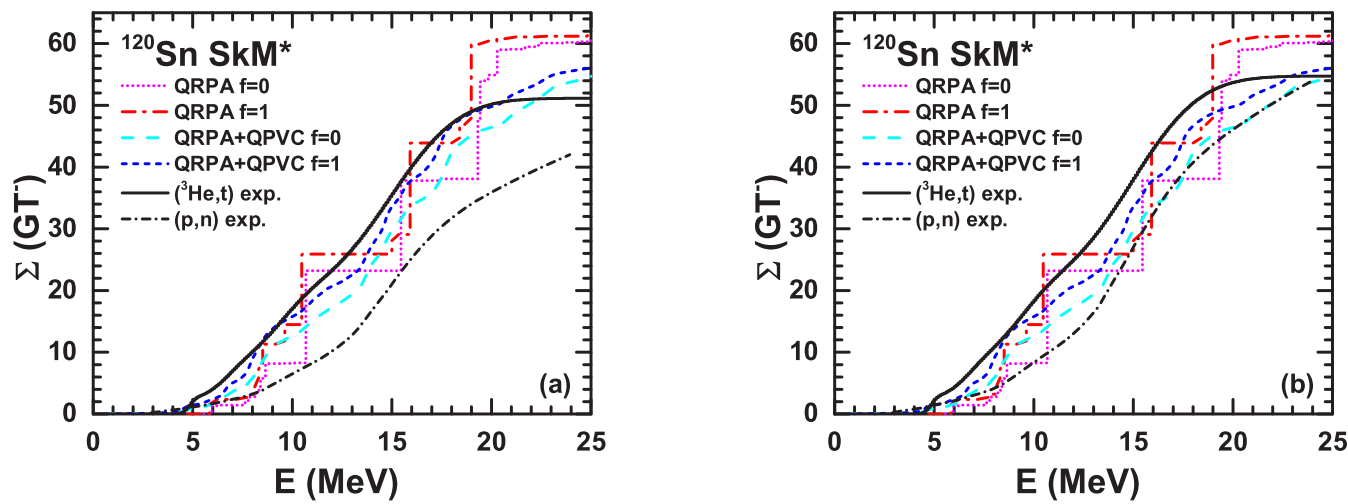

FIG. 10. The cumulative sum of Gamow-Teller strength for ${ }^{120} \mathrm{Sn}$, calculated by QRPA and QRPA+QPVC models, with and without isoscalar pairing, using the Skyrme interaction SkM*. The experimental results from $\left({ }^{3} \mathrm{He}, t\right)[53]$ and $(p, n)$ reactions [54] are shown for comparison. In panel (a), the $B(\mathrm{GT})$ strength from the $\left({ }^{3} \mathrm{He}, t\right)$ experiment is obtained by multiplying the cross section by a factor of 1.6 so that the main GTR strength exhausts $65 \%$ of the Ikeda sum rule, while the $B(\mathrm{GT})$ strength for the $(p, n)$ experiment is obtained by dividing the cross section by the unit cross section. In panel (b), the two experimental cumulative GT sums are normalized to the same value as the theoretical one of QRPA+QPVC with $f=0$ at $E=25 \mathrm{MeV}$. 
TABLE IV. Microscopic structure of the main GT peaks found above $E=13 \mathrm{MeV}$ in the QRPA+QPVC calculations with IS pairing $(f=1.0)$, shown in Fig. 9(b). We list the peak energy $E$, the complex eigenenergy $\Omega_{v}-i \frac{\Gamma_{v}}{2}$ from QRPA+QPVC, the energy $E_{m}$, and the forward amplitudes $X_{a b}^{(m)}$ of the associated QRPA state $|m\rangle$ (cf. Table III), the imaginary part of the diagonal spreading matrix element $W_{a b, a b}$, and the contributions to the imaginary part of the self-energy $\mathcal{A}_{1}$.

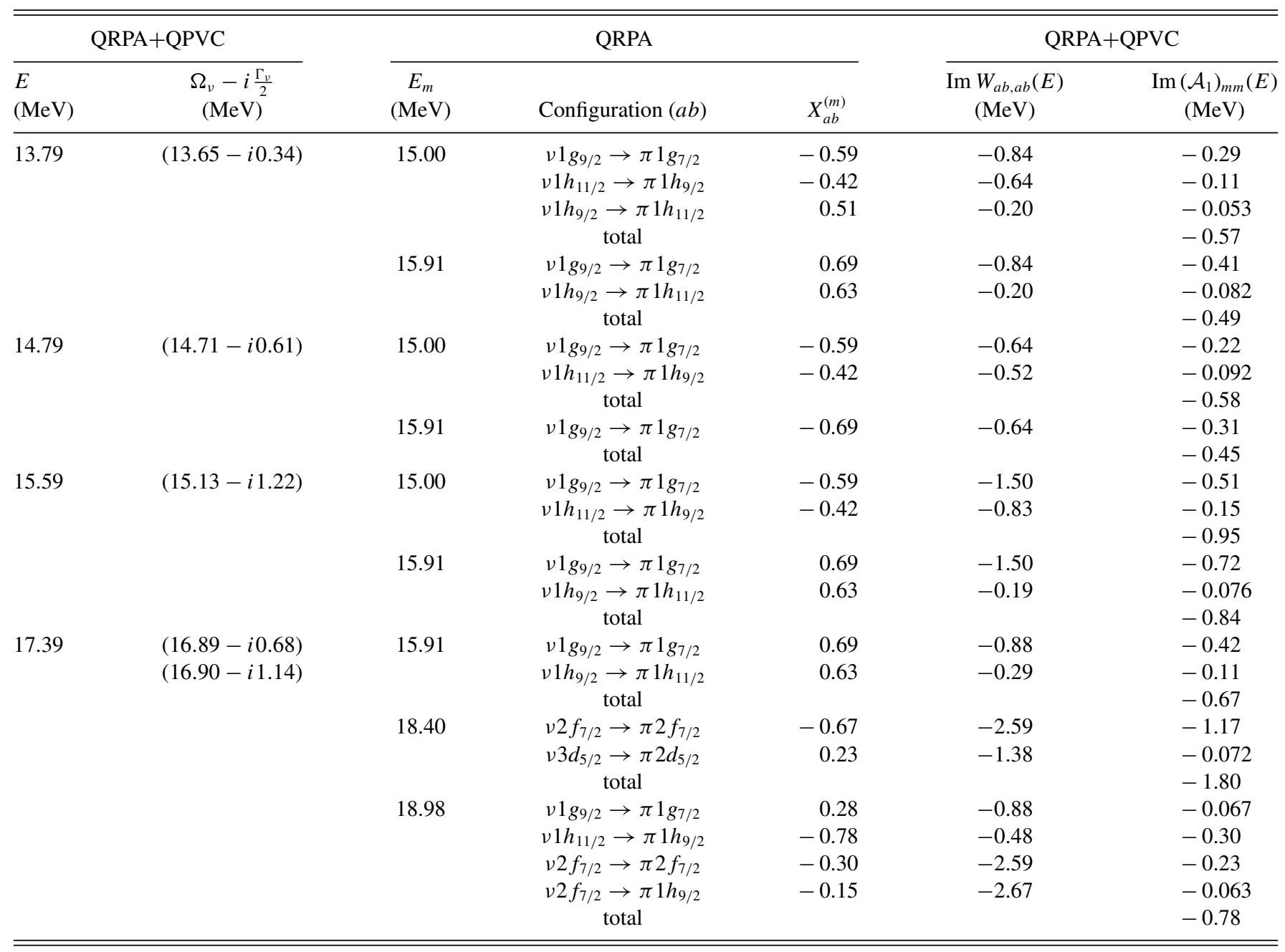

are also plotted with respect to the mother nucleus in the figures.

The resulting cumulative $B(\mathrm{GT})$ is shown in Fig. 10(a). The results of these two experiments are quite different in the low-energy region and therefore also in the total strength up to $E=25 \mathrm{MeV}$. In Ref. [53], it is stated that the $B(\mathrm{GT})$ values that have been extracted exceed the expectations for the low-lying strength; in particular, in the case of ${ }^{118,120} \mathrm{Sb}$ the strength exceeds what can be deduced from the known $\log (f t)$ values of the $\beta^{+}$decay, by about a factor of 5 . The authors concluded that this is because only $20 \%$ of the observed $\left({ }^{3} \mathrm{He}, t\right)$ charge-exchange transition strength is due to $\Delta L=0$ spin-flip transition mediated by the central interaction $V_{\sigma \tau}$, while about $80 \%$ is due to $\Delta L=2$ spin-flip transition mediated by the noncentral tensor interaction $V_{T \tau}$. One example of such a $\Delta L=2$ spin-flip transition is the particle-hole configuration $\left(2 d_{5 / 2}\right)\left(1 g_{7 / 2}\right)^{-1}$ which will not be excited by the GT operator. Therefore, a quantitative comparison between the $\left({ }^{3} \mathrm{He}, t\right)$ data and the theoretical prediction for the $B(G T)$ values is not straightforward. On the other hand, the results of the $(p, n)$ reaction are dominated by $\Delta L=0$ spin-flip transitions and the comparison with the theoretical $B(\mathrm{GT})$ strength is more realistic. Nevertheless, we should keep in mind that our extraction of the $B(\mathrm{GT})$ strength from the $(p, n)$ data is approximated, and a detailed analysis of the kinetic correction factor $F(q, \omega)$ would be required to obtain more quantitative $B(\mathrm{GT})$ data.

To compare better the profile of the cumulative sum, we normalize the experimental cumulative sums to the theoretical value of QRPA+QPVC with $f=0$ at $E=25 \mathrm{MeV}$, and plot them in Fig. 10(b). At the QRPA level, the low-energy strength is increased going from $f=0$ to $f=1$, while the total strength at $E=25 \mathrm{MeV}$ is almost the same, and close to $3(N-Z)$. The development of the spreading width substantially improves the comparison with experiment when going from the QRPA to QRPA+QPVC. Going from $f=0$ to $f=1$, the empirical low-energy strength is increased, so the $f=1$ result is closer to the $\left({ }^{3} \mathrm{He}, t\right)$ experiment. The $f=0$ result is very close to the $(p, n)$ experiment, although it still overestimates the low-lying strength. Substantial quenching 

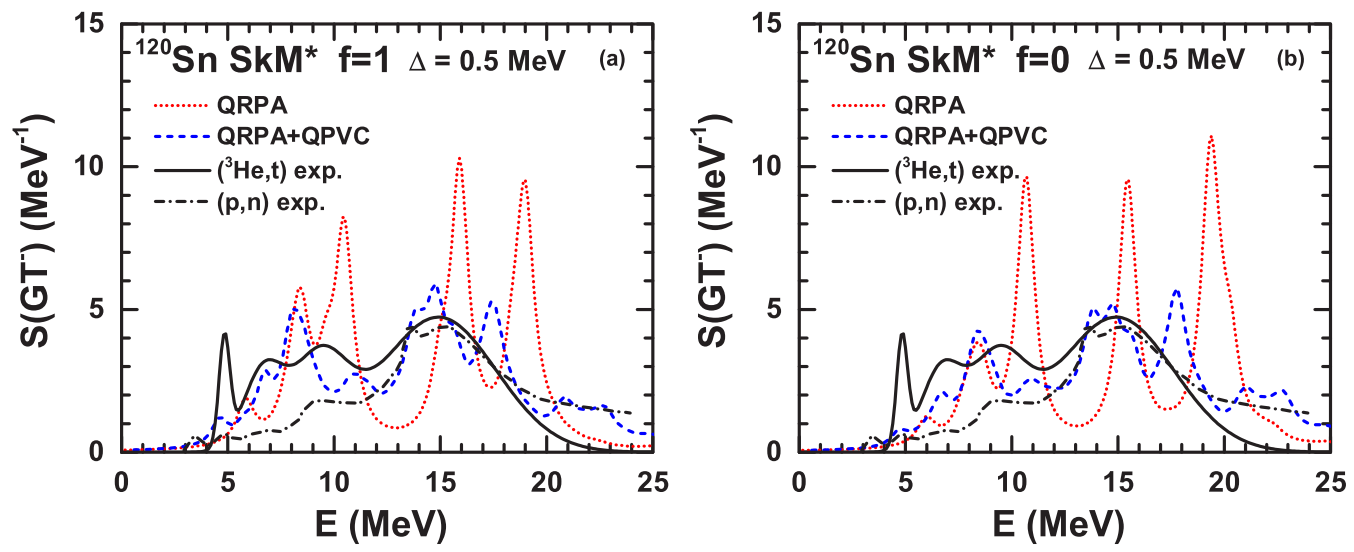

FIG. 11. The Gamow-Teller strength distributions for ${ }^{120} \mathrm{Sn}$ calculated by QRPA and QRPA+QPVC models, with [(a) and without (b) isoscalar pairing, using the Skyrme interaction $\mathrm{SkM}^{*}$. The smearing parameter $\Delta=0.5 \mathrm{MeV}$ is used instead of $\Delta=0.2 \mathrm{MeV}$ used for Fig. 9 . The experimental results from $\left({ }^{3} \mathrm{He}, t\right)$ and $(p, n)$ reactions are shown for comparison. The cross section from the $\left({ }^{3} \mathrm{He}, t\right)$ experiment is scaled by a factor of 1.6 so that the main GTR strength exhausts $65 \%$ of Ikeda sum rule [53]. The cross section from the $(p, n)$ reaction is normalized by the unit cross section [54] (cf. the main text).

in the $(p, n)$ data is found in the cumulative sum of $B\left(\mathrm{GT}^{-}\right)$ strength up to $E=24 \mathrm{MeV}$, which exhausts only $70 \%$ of the GT Ikeda sum rule. With the inclusion of the QPVC effect, the calculated strength up to $E=25 \mathrm{MeV}$ is about the same without and with the IS pairing, i.e., $f=0$ or $f=1$, and it is quenched by about $10 \%$ with respect to the QRPA results. It turns out that the experimental cumulative sum of $B\left(\mathrm{GT}^{-}\right)$strength up to $E=24 \mathrm{MeV}$ is about $75 \%$ of the QRPA+QPVC result, which is consistent with our previous investigation on the quenching problem for ${ }^{208} \mathrm{~Pb}$ using the RPA+PVC model [30]. The QRPA+QPVC model improves the description of the quenching problem compared to the QRPA model by around $10 \%$, and the remaining discrepancies with experimental data may originate from various sources such as the $\Delta$-isobar excitation, the tensor force, the deficiencies of our model (e.g., the omission of high-energy $2 \mathrm{p}-2 \mathrm{~h}$ doorway states) or systematic uncertainties of the experimental analysis.

The four theoretical strength functions are compared with experiment in Fig. 11. We use a smearing parameter $\Delta=$ $0.5 \mathrm{MeV}$ in the QRPA and QRPA+QPVC calculation, instead of the value $\Delta=0.2$ previously used in Fig. 9. This value corresponds to the energy resolution of the $(p, n)$ experiment. As in Fig. 10, the $\left({ }^{3} \mathrm{He}, t\right)$ experimental low-energy strength distribution is well reproduced by including isoscalar pairing, while the $(p, n)$ data are better reproduced without it. The spreading width and lineshape of the giant resonance region are very well reproduced by the inclusion of the QPVC effect.

\section{SUMMARY AND PERSPECTIVES}

The self-consistent QRPA+QPVC model based on Skyrme density functionals has been developed for the first time and applied to the calculation of the GT strength distribution of the superfluid nucleus ${ }^{120} \mathrm{Sn}$. This model is an extension of the previously developed RPA+PVC model for magic nuclei, yet with specific features that have been discussed in detail in this work, starting from the inclusion of isoscalar pairing. Moreover, the subtraction method has been adopted and its impact on the results has been elucidated. We have mainly discussed the results obtained by using the $\mathrm{SkM}^{*}$ force, which gives the best description among the three Skyrme forces we have considered, consistently with previous results in nonsuperfluid nuclei [29-31].

Specifically, we studied the effect of the attractive isoscalar pairing interaction on GT states in QRPA and QRPA+QPVC models, respectively. In both models, it slightly shifts the low-lying strength distribution downwards and increases its amount in the low-energy region. The inclusion of QPVC on top of simple QRPA produces a conspicuous spreading width and is quite relevant to reproduce well the experimental line shape of the strength distribution. As an overall effect, several peaks that are found in the QRPA model to lie in the giant resonance region are merged into one big resonance peak with four subpeaks in our calculation. The microscopic structure as well as the origin of the widths of these four subpeaks were analyzed in detail. The cumulative GT strength distribution was compared with the experimental data. Our QRPA+QPVC result reproduces very well the $(p, n)$ data without the inclusion of isoscalar pairing, except for a slight overestimate of the low-lying strength.

The inclusion of pairing correlations paves the way to many possible applications of our model to charge-exchange transitions in the case of nuclei far from stability line. In fact, the HFB plus QRPA is the appropriate tool for these neutron-rich, or neutron-deficient, nuclei, especially for weakly bound nuclei. Charge-exchange reactions or $\beta$ decay are valid spectroscopic tools for these nuclei, but mean-field or DFT calculations cannot describe the damping width due to the lack of coupling with more complicated configurations, and they also tend to overestimate the $\beta$-decay half-lives when applied to such processes in exotic nuclei. Benchmarking PVC calculations in these cases is a new research line which is still in its infancy. Improving the theoretical predictive power of such calculations is not only beneficial for our progress in understanding nuclear structure, but also weakinteraction processes are of essential interest for particle physics or astrophysics. Accordingly, we envisage the study of 
weak-interaction processes of astrophysical interest in our future research of the QRPA+QPVC model.

\section{ACKNOWLEDGMENTS}

This work was partly supported by the National Natural Science Foundation of China under Grant No. 11305161 and by JSPS KAKENHI Grant No. JP16K05367. Funding from the European Unions Horizon 2020 research and innovation program under Grant Agreement No. 654002 is also acknowledged.

\section{APPENDIX A: THE CALCULATION OF $\langle a b|V| N\rangle$}

We wish to calculate

$$
\langle a b|V| N\rangle=\left\langle 0\left|\alpha_{b} \alpha_{a} V \alpha_{a^{\prime \prime}}^{\dagger} \alpha_{b^{\prime \prime}}^{\dagger} \Gamma_{n L}^{\dagger}\right| 0\right\rangle,
$$

with

$$
\Gamma_{n L}^{\dagger}=\frac{1}{\sqrt{1+\delta_{c d}}} \sum_{c \geqslant d} X_{c d} \alpha_{c}^{\dagger} \alpha_{d}^{\dagger}-Y_{c d} \alpha_{d} \alpha_{c} .
$$

Since $|0\rangle$ is the vacuum for HFB quasiparticle states, we transform the BCS quasiparticle states $|a\rangle$, associated with the operators $\alpha_{a}^{\dagger}$, to HFB quasiparticle states $|\tilde{a}\rangle$, associated with the operators $\beta_{\tilde{a}}^{\dagger}$, based on the following unitary transformation [42]:

$$
\begin{aligned}
& \alpha_{a}=\sum_{\tilde{a}} C_{a \tilde{a}} \beta_{\tilde{a}}, \\
& \alpha_{a}^{\dagger}=\sum_{\tilde{a}} C_{a \tilde{a}}^{*} \beta_{\tilde{a}}^{\dagger} .
\end{aligned}
$$

We will have

$$
\langle a b|V| N\rangle=\sum_{\tilde{a} \tilde{\tilde{a}} \tilde{a}^{\prime \prime} \tilde{b}^{\prime \prime}} C_{a \tilde{a}} C_{b \tilde{b}} C_{a^{\prime \prime} \tilde{a}^{\prime \prime}}^{*} C_{b^{\prime \prime} \tilde{b}^{\prime \prime}}^{*}\left\langle 0\left|\beta_{\tilde{b}} \beta_{\tilde{a}} V \beta_{\tilde{a}^{\prime \prime}}^{\dagger} \beta_{\tilde{b}^{\prime \prime}}^{\dagger} \Gamma_{n L}^{\dagger}\right| 0\right\rangle
$$

and

$$
\begin{aligned}
\Gamma_{n L}^{\dagger}= & \frac{1}{\sqrt{1+\delta_{c d}}} \sum_{c \geqslant d} \sum_{\tilde{c} \tilde{d}} C_{c \tilde{c}}^{*} C_{d \tilde{d}}^{*} X_{c d} \beta_{\tilde{c}}^{\dagger} \beta_{\tilde{d}}^{\dagger} \\
& -C_{c \tilde{c}} C_{d \tilde{d}} Y_{c d} \beta_{\tilde{d}} \beta_{\tilde{c}} .
\end{aligned}
$$

In the case of QRPA phonons, we make the following approximation:

$$
\left\langle 0\left|\beta_{\tilde{b}} \beta_{\tilde{a}} V \beta_{\tilde{a}^{\prime \prime}}^{\dagger} \beta_{\tilde{b}^{\prime \prime}}^{\dagger} \Gamma_{n L}^{\dagger}\right| 0\right\rangle \simeq\left\langle 0\left|\beta_{\tilde{b}} \beta_{\tilde{a}}\left[V, \Gamma_{n L}^{\dagger}\right] \beta_{\tilde{a}^{\prime \prime}}^{\dagger} \beta_{\tilde{b}^{\prime \prime}}^{\dagger}\right| 0\right\rangle
$$

Then we obtain

$$
\begin{aligned}
\langle a b|V| N\rangle= & \frac{1}{\sqrt{1+\delta_{c d}}} \sum_{\tilde{a} \tilde{b} \tilde{a}^{\prime \prime} \tilde{b}^{\prime \prime}} C_{a \tilde{a}} C_{b \tilde{b}} C_{a^{\prime \prime} \tilde{a}^{\prime \prime}}^{*} C_{b^{\prime \prime} \tilde{b}^{\prime \prime}}^{*} \sum_{c \geqslant d} \sum_{\tilde{c} \tilde{d}}\left[C_{c \tilde{c} \tilde{c}}^{*} C_{d \tilde{d}}^{*}\left\langle 0\left|\beta_{\tilde{b}} \beta_{\tilde{a}} V \beta_{\tilde{a}^{\prime \prime}}^{\dagger} \beta_{\tilde{b}^{\prime \prime}}^{\dagger} \beta_{\tilde{c}}^{\dagger} \beta_{\tilde{d}}^{\dagger}\right| 0\right\rangle X_{c d}\right. \\
& \left.+C_{c \tilde{c}} C_{d \tilde{d}}\left\langle 0\left|\beta_{\tilde{b}} \beta_{\tilde{a}} \beta_{\tilde{d}} \beta_{\tilde{c}} V \beta_{\tilde{a}^{\prime \prime}}^{\dagger} \beta_{\tilde{b}^{\prime \prime}}^{\dagger}\right| 0\right\rangle Y_{c d}\right] .
\end{aligned}
$$

Here $V$ is the two-body interaction for the coupling vertex. It has the general form, in the single-particle basis,

$$
V=\frac{1}{4} \sum_{1234} V_{1234} c_{1}^{\dagger} c_{2}^{\dagger} c_{4} c_{3}
$$

and can be written in the HFB quasiparticle basis

$$
V=\sum_{\tilde{a} \tilde{b} \tilde{c} \tilde{d}}\left(H_{\tilde{a} \tilde{b} \tilde{c} \tilde{d}}^{40} \beta_{\tilde{a}}^{\dagger} \beta_{\tilde{b}}^{\dagger} \beta_{\tilde{c}}^{\dagger} \beta_{\tilde{d}}^{\dagger}+\text { H.c. }\right)+\sum_{\tilde{a} \tilde{b} \tilde{c} \tilde{d}}\left(H_{\tilde{a} \tilde{b} \tilde{c} \tilde{d}}^{31} \beta_{\tilde{a}}^{\dagger} \beta_{\tilde{b}}^{\dagger} \beta_{\tilde{c}}^{\dagger} \beta_{\tilde{d}}+\text { H.c. }\right)+\frac{1}{4} \sum_{\tilde{a} \tilde{b} \tilde{c} \tilde{d}} H_{\tilde{a} \tilde{b} \tilde{c} \tilde{d}}^{22} \beta_{\tilde{a}}^{\dagger} \beta_{\tilde{b}}^{\dagger} \beta_{\tilde{d}} \beta_{\tilde{c}} \text {. }
$$

Using the Wick theorem, only the $H^{31}$ or $H^{13}$ terms exist in $\langle a b|V| N\rangle$, and we get

$$
\begin{aligned}
& \langle a b|V| N\rangle=\frac{1}{\sqrt{1+\delta_{c d}}} \sum_{\tilde{a} \tilde{b} \tilde{a}^{\prime \prime} \tilde{b}^{\prime \prime}} C_{a \tilde{a}} C_{b \tilde{b}} C_{a^{\prime \prime} \tilde{a}^{\prime \prime}}^{*} C_{b^{\prime \prime} \tilde{b}^{\prime \prime}}^{*} \sum_{c \geqslant d} \sum_{\tilde{c} \tilde{d}} C_{c \tilde{c}}^{*} C_{d \tilde{d}}^{*}\left[\delta_{\tilde{b} \tilde{b}^{\prime \prime}}\left(H_{\tilde{a}^{\prime \prime} \tilde{c} \tilde{d} \tilde{a}}^{13}-H_{\tilde{a}^{\prime \prime} \tilde{d} \tilde{a} \tilde{a}}^{13}+H_{\tilde{c} \tilde{d} \tilde{a}^{\prime \prime} \tilde{a}}^{13}-H_{\tilde{c} \tilde{a}^{\prime \prime} \tilde{d} \tilde{a}}^{13}+H_{\tilde{d} \tilde{a}^{\prime \prime} \tilde{c} \tilde{a}}^{13}-H_{\tilde{d} \tilde{c} \tilde{a}^{\prime \prime} \tilde{a}}^{13}\right) X_{c d}\right. \\
& \left.+\delta_{\tilde{a} \tilde{a}^{\prime \prime}}\left(H_{\tilde{b}^{\prime \prime} \tilde{c} \tilde{d} \tilde{b}}^{13}-H_{\tilde{b}^{\prime \prime} \tilde{d} \tilde{c} \tilde{b}}^{13}+H_{\tilde{c} \tilde{d} \tilde{b}^{\prime \prime} \tilde{b}}^{13}-H_{\tilde{c} \tilde{b}^{\prime \prime} \tilde{d} \tilde{b}}^{13}+H_{\tilde{d} \tilde{b}^{\prime \prime} \tilde{c} \tilde{b}}^{13}-H_{\tilde{d} \tilde{c} \tilde{b}^{\prime \prime} \tilde{b}}^{13}\right) X_{c d}\right] \\
& +C_{c \tilde{c}} C_{d \tilde{d}}\left[\delta_{\tilde{b} \tilde{b}^{\prime \prime}}\left(H_{\tilde{a} \tilde{c} \tilde{c} \tilde{a} \tilde{a}^{\prime \prime}}^{31}-H_{\tilde{a} \tilde{d} \tilde{c} \tilde{a}^{\prime \prime}}^{31}+H_{\tilde{c} \tilde{d} \tilde{a} \tilde{a}^{\prime \prime}}^{31}-H_{\tilde{c} \tilde{a} \tilde{a} \tilde{a}^{\prime \prime}}^{31}+H_{\tilde{d} \tilde{a} \tilde{a} \tilde{a}^{\prime \prime}}^{31}-H_{\tilde{d} \tilde{c} \tilde{a} \tilde{a}^{\prime \prime}}^{31}\right) Y_{c d}\right. \\
& \left.+\delta_{\tilde{a} \tilde{a} \tilde{a}^{\prime \prime}}\left(H_{\tilde{b} \tilde{c} \tilde{c} \tilde{b}^{\prime \prime}}^{31}-H_{\tilde{b} \tilde{c} \tilde{c} \tilde{b}^{\prime \prime}}^{31}+H_{\tilde{c} \tilde{d} \tilde{b} \tilde{b}^{\prime \prime}}^{31}-H_{\tilde{c} \tilde{b} \tilde{b} \tilde{b}^{\prime \prime}}^{31}+H_{\tilde{d} \tilde{c} \tilde{b} \tilde{b}^{\prime \prime}}^{31}-H_{\tilde{d} \tilde{c} \tilde{b} \tilde{b}^{\prime \prime}}^{31}\right) Y_{c d}\right] \\
& =\frac{1}{\sqrt{1+\delta_{c d}}} \sum_{\tilde{a} \tilde{b} \tilde{a}^{\prime \prime} \tilde{b}^{\prime \prime}} C_{a \tilde{a}} C_{b \tilde{b}} C_{a^{\prime \prime} \tilde{a}^{\prime \prime}}^{*} C_{b^{\prime \prime} \tilde{b}^{\prime \prime}}^{*} \sum_{c \geqslant d} \sum_{\tilde{c} \tilde{d}} C_{c \tilde{c}}^{*} C_{d \tilde{d}}^{*}\left[\delta_{\tilde{b} \tilde{b}^{\prime \prime}} 2\left(H_{\tilde{a}^{\prime \prime} \tilde{c} \tilde{a} \tilde{a}}^{13}+H_{\tilde{c} \tilde{d} \tilde{a}^{\prime \prime} \tilde{a}}^{13}+H_{\tilde{d} \tilde{a}^{\prime \prime} \tilde{c} \tilde{a}}^{13}\right) X_{c d}\right. \\
& \left.+\delta_{\tilde{a} \tilde{a}^{\prime \prime}} 2\left(H_{\tilde{b}^{\prime \prime} \tilde{c} \tilde{b} \tilde{b}}^{13}+H_{\tilde{c} \tilde{d} \tilde{b}^{\prime \prime} \tilde{b}}^{13}+H_{\tilde{d} \tilde{b}^{\prime \prime} \tilde{c} \tilde{b}}^{13}\right) X_{c d}\right]+C_{c \tilde{c}} C_{d \tilde{d}}\left[\delta_{\tilde{b} \tilde{b}^{\prime \prime}} 2\left(H_{\tilde{a} \tilde{c} \tilde{d} \tilde{a}^{\prime \prime}}^{31}+H_{\tilde{c} \tilde{d} \tilde{a} \tilde{a}^{\prime \prime}}^{31}+H_{\tilde{d} \tilde{a} \tilde{c} \tilde{a}^{\prime \prime}}^{31}\right) Y_{c d}\right. \\
& \left.+\delta_{\tilde{a} \tilde{a} \tilde{a}^{\prime \prime}}\left(H_{\tilde{b} \tilde{c} \tilde{d} \tilde{b}^{\prime \prime}}^{31}+H_{\tilde{c} \tilde{d} \tilde{b} \tilde{b}^{\prime \prime}}^{31}+H_{\tilde{d} \tilde{b} \tilde{c} \tilde{b}^{\prime \prime}}^{31}\right) Y_{c d}\right] .
\end{aligned}
$$


With the $C$ matrix, $H_{\tilde{a} \tilde{c} \tilde{c} \tilde{d}}^{31}$ on HFB quasiparticle states can be transformed to $H_{a b c d}^{31}$ on BCS quasiparticle states, so that

$$
\begin{aligned}
\langle a b|V| N\rangle= & \frac{1}{\sqrt{1+\delta_{c d}}} \sum_{c \geqslant d}\left[\delta_{b b^{\prime \prime}} 2\left(H_{a^{\prime \prime} c d a}^{13}+H_{c d a^{\prime \prime} a}^{13}+H_{d a^{\prime \prime} c a}^{13}\right) X_{c d}+\delta_{a a^{\prime \prime}} 2\left(H_{b^{\prime \prime} c d b}^{13}+H_{c d b^{\prime \prime} b}^{13}+H_{d b^{\prime \prime} c b}^{13}\right) X_{c d}\right] \\
& +\left[\delta_{b b^{\prime \prime}} 2\left(H_{a c d a^{\prime \prime}}^{31}+H_{c d a a^{\prime \prime}}^{31}+H_{d a c a^{\prime \prime}}^{31}\right) Y_{c d}+\delta_{a a^{\prime \prime}} 2\left(H_{b c d b^{\prime \prime}}^{31}+H_{c d b b^{\prime \prime}}^{31}+H_{d b c b^{\prime \prime}}^{31}\right) Y_{c d}\right] .
\end{aligned}
$$

From Ref. [42] we know that

$$
H_{\tilde{a} \tilde{b} \tilde{c} \tilde{d}}^{31}=\frac{1}{2} \sum_{1234} V_{1234}\left[U_{1 \tilde{a}}^{*} V_{4 \tilde{b}}^{*} V_{3 \tilde{c}}^{*} V_{2 \tilde{d}}+V_{3 \tilde{a}}^{*} U_{2 \tilde{b}}^{*} U_{1 \tilde{c}}^{*} U_{4 \tilde{d}}\right]
$$

where $\tilde{a}, \tilde{b}, \tilde{c}, \tilde{d}$ denote the states of the quasiparticle basis, and 1,2,3,4 denote the states of the single-particle basis. After transformation with $C, H_{\tilde{a} \tilde{c} \tilde{c} \tilde{d}}^{31}$ becomes $H_{a b c d}^{31}$ on the BCS quasiparticle basis with the form

$$
H_{a b c d}^{31}=\frac{1}{2} \sum_{\tilde{1} \tilde{2} \tilde{3} \tilde{4}} V_{\tilde{1} \tilde{2} \tilde{3} \tilde{4}}\left[\bar{U}_{\tilde{1} a}^{*} \bar{V}_{\tilde{4} b}^{*} \bar{V}_{\tilde{3} c}^{*} \bar{V}_{\tilde{2} d}+\bar{V}_{\tilde{3} a}^{*} \bar{U}_{\tilde{2} b}^{*} \bar{U}_{\tilde{1} c}^{*} \bar{U}_{\tilde{4} d}\right]
$$

where $\tilde{1}, \tilde{2}, \tilde{3}, \tilde{4}$ denote the canonical basis. The $\bar{U}$ and $\bar{V}$ matrices connect the canonical basis and BCS quasiparticle basis, and their definition is found in Ref. [42]. The $\bar{U}$ and $\bar{V}$ matrices can be further simplified as

$$
\bar{U}_{\tilde{1} a}=u_{\tilde{1}} \delta_{\tilde{1} a}, \quad \bar{V}_{\tilde{1} a}=-v_{\tilde{1}} \delta_{\tilde{1} \tilde{a}},
$$

where $u, v$ denote the occupation amplitudes in the canonical basis. Then

$$
\begin{aligned}
& H^{31}=\sum_{a b c d} H_{a b c d}^{31} \alpha_{a}^{\dagger} \alpha_{b}^{\dagger} \alpha_{c}^{\dagger} \alpha_{d}=\sum_{a b c d} \frac{1}{2} \sum_{\tilde{1} \tilde{2} \tilde{3} \tilde{4}} V_{\tilde{1} \tilde{2} \tilde{3} \tilde{4}}\left[\bar{U}_{\tilde{1} a}^{*} \bar{V}_{\tilde{4} b}^{*} \bar{V}_{\tilde{3} c}^{*} \bar{V}_{\tilde{2} d}+\bar{V}_{\tilde{3} a}^{*} \bar{U}_{\tilde{2} b}^{*} \bar{U}_{\tilde{1} c}^{*} \bar{U}_{\tilde{4} d}\right] \alpha_{a}^{\dagger} \alpha_{b}^{\dagger} \alpha_{c}^{\dagger} \alpha_{d} \\
& =-\sum_{a b c d} \frac{1}{2} \sum_{\tilde{1} \tilde{2} \tilde{3} \tilde{4}} V_{\tilde{1} \tilde{2} \tilde{3} \tilde{4}}\left(u_{\tilde{1}} v_{\tilde{2}} v_{\tilde{3}} v_{\tilde{4}} \delta_{\tilde{1} a} \delta_{\tilde{2} \tilde{d}} \delta_{\tilde{3} \bar{c}} \delta_{\tilde{4} \tilde{b}} \alpha_{\tilde{1}}^{\dagger} \alpha_{\tilde{\tilde{4}}}^{\dagger} \alpha_{\tilde{\tilde{3}}}^{\dagger} \alpha_{\tilde{2}}+u_{\tilde{1}} u_{\tilde{2}} v_{\tilde{3}} u_{4} \delta_{\tilde{1} c} \delta_{\tilde{2} b} \delta_{\tilde{3} \tilde{a}} \delta_{\tilde{4} d} \alpha_{\tilde{\tilde{z}}}^{\dagger} \alpha_{\tilde{2}}^{\dagger} \alpha_{\tilde{1}}^{\dagger} \alpha_{\tilde{4}}\right) \\
& =\sum_{a b c d} \frac{1}{2} \sum_{\tilde{1} \tilde{2} \tilde{3} \tilde{4}} V_{\tilde{4} \tilde{3} \tilde{3} \tilde{2}}\left[u_{\tilde{1}} u_{\tilde{2}} v_{\tilde{3}} u_{\tilde{4}}-v_{\tilde{1}} v_{\tilde{2}} u_{\tilde{3}} v_{\tilde{4}}\right] \alpha_{\tilde{1}}^{\dagger} \alpha_{\tilde{2}}^{\dagger} \alpha_{\tilde{3}}^{\dagger} \alpha_{\tilde{4}} \delta_{\tilde{1} a} \delta_{\tilde{2} b} \delta_{\tilde{3} c} \delta_{\tilde{4} d} \\
& =\frac{1}{2} \sum_{\tilde{1} \tilde{2} \tilde{3} \tilde{4}} V_{\tilde{4} \tilde{3} \tilde{1} \tilde{2}}\left[u_{\tilde{1}} u_{\tilde{2}} v_{\tilde{3}} u_{\tilde{4}}-v_{\tilde{1}} v_{\tilde{2}} u_{\tilde{3}} v_{\tilde{4}}\right] \alpha_{\tilde{1}}^{\dagger} \alpha_{\tilde{2}}^{\dagger} \alpha_{\tilde{3}}^{\dagger} \alpha_{\tilde{4}} \\
& =\frac{1}{2} \sum_{a b c d} V_{d \bar{c} a b}\left[u_{a} u_{b} v_{c} u_{d}-v_{a} v_{b} u_{c} v_{d}\right] \alpha_{a}^{\dagger} \alpha_{b}^{\dagger} \alpha_{c}^{\dagger} \alpha_{d} .
\end{aligned}
$$

Finally we can write $H_{a b c d}^{31}$ in the canonical basis,

$$
H_{a b c d}^{31}=\frac{1}{2} V_{d \bar{c} a b}\left[u_{a} u_{b} v_{c} u_{d}-v_{a} v_{b} u_{c} v_{d}\right] .
$$

Similarly,

$$
H_{a b c d}^{13}=\frac{1}{2} V_{d \bar{c} a b}^{*}\left[u_{a} u_{b} v_{c} u_{d}-v_{a} v_{b} u_{c} v_{d}\right] .
$$

Combining the above Eq. (A20) and Eq. (A21) with Eq. (A13), one arrives at Eq. (12) and (13) in Sec. II.

\section{APPENDIX B: ANGULAR MOMENTUM COUPLED FORM}

In Eq. (A13), the term $V\left(a a^{\prime \prime} c d\right)$ in front of $X$ can be expressed in angular momentum coupled form,

$$
V\left(a a^{\prime \prime} c d\right)=\left\langle j_{c} m_{c} j_{d} m_{d} \mid L M\right\rangle\left\langle j_{a} m_{a} j_{a^{\prime \prime}}-m_{a^{\prime \prime}} \mid L M\right\rangle(-1)^{j_{a^{\prime \prime}}-m_{a^{\prime \prime}}} \frac{\hat{L}}{\hat{j}_{a}} V\left(c d L a^{\prime \prime} ; a\right),
$$

where

$$
\begin{aligned}
V\left(c d L a^{\prime \prime} ; a\right) & =\sum_{m_{c} m_{d} m_{a^{\prime \prime}} m_{a}}\left\langle j_{c} m_{c} j_{d} m_{d} \mid L M\right\rangle\left\langle j_{a^{\prime \prime}} m_{a^{\prime \prime}} L M \mid j_{a} m_{a}\right\rangle V\left(a a^{\prime \prime} c d\right) \\
& =\sum_{m_{c} m_{d} m_{a^{\prime \prime}} m_{a}}\left\langle j_{c} m_{c} j_{d} m_{d} \mid L M\right\rangle\left\langle j_{a} m_{a} j_{a^{\prime \prime}}-m_{a^{\prime \prime}} \mid L M\right\rangle(-1)^{j_{a^{\prime \prime}}-m_{a^{\prime \prime}}} \frac{\hat{j} a}{\hat{L}} V\left(a a^{\prime \prime} c d\right) .
\end{aligned}
$$


Calculating the three matrix elements in $V\left(a a^{\prime \prime} c d\right)$ with the Clebsch-Gordan coefficients, we finally get

$$
\begin{aligned}
V\left(c d L a^{\prime \prime} ; a\right)= & \frac{\hat{j}_{a}}{\hat{L}}\left[V_{a d a^{\prime \prime} c}^{L p h}\left(u_{a^{\prime \prime}} u_{c} v_{d} u_{a}-v_{a^{\prime \prime}} v_{c} u_{d} v_{a}\right)+V_{a c a^{\prime \prime} d}^{L p h}\left(u_{d} u_{a^{\prime \prime}} v_{c} u_{a}-v_{d} v_{a^{\prime \prime}} u_{c} v_{a}\right)(-1)^{j_{c}-j_{d}+L}\right. \\
& \left.-V_{a a^{\prime \prime} c d}^{L p p}\left(u_{c} u_{d} v_{a^{\prime \prime}} u_{a}-v_{c} v_{d} u_{a^{\prime \prime}} v_{a}\right)\right] \\
\equiv & \widetilde{V}\left(c d L a^{\prime \prime} ; a\right) \frac{\hat{j}_{a}}{\hat{L}} .
\end{aligned}
$$

Similarly, the term in front of $Y$ is

$$
V\left(a^{\prime \prime} a c d\right)=\left\langle j_{c} m_{c} j_{d} m_{d} \mid L-M\right\rangle(-1)^{L-M}\left\langle j_{a} m_{a} j_{a^{\prime \prime}}-m_{a^{\prime \prime}} \mid L M\right\rangle(-1)^{j_{a^{\prime \prime}}-m_{a^{\prime \prime}}} \frac{\hat{L}}{\hat{j}_{a}} V\left(c d L a ; a^{\prime \prime}\right),
$$

where

$$
\begin{aligned}
V\left(c d L a ; a^{\prime \prime}\right) & =\sum_{m_{c} m_{d} m_{a^{\prime \prime}} m_{a}}\left\langle j_{c} m_{c} j_{d} m_{d} \mid L-M\right\rangle(-1)^{L-M}\left\langle j_{a^{\prime \prime}} m_{a^{\prime \prime}} L M \mid j_{a} m_{a}\right\rangle V\left(a^{\prime \prime} a c d\right) \\
& =\sum_{m_{c} m_{d} m_{a^{\prime \prime}} m_{a}}\left\langle j_{c} m_{c} j_{d} m_{d} \mid L-M\right\rangle(-1)^{L-M}\left\langle j_{a} m_{a} j_{a^{\prime \prime}}-m_{a^{\prime \prime}} \mid L M\right\rangle(-1)^{j_{a^{\prime \prime}}-m_{a^{\prime \prime}}} \frac{\hat{j_{a}}}{\hat{L}} V\left(a^{\prime \prime} a c d\right) .
\end{aligned}
$$

We have

$$
\begin{aligned}
V\left(c d L a ; a^{\prime \prime}\right)= & {\left[V_{a^{\prime \prime} d a c}^{L p h}\left(u_{a^{\prime \prime}} u_{c} v_{d} u_{a}-v_{a^{\prime \prime}} v_{c} u_{d} v_{a}\right)+V_{a^{\prime \prime} c a d}^{L p h}\left(u_{d} u_{a^{\prime \prime}} v_{c} u_{a}-v_{d} v_{a^{\prime \prime}} u_{c} v_{a}\right)(-1)^{j_{c}-j_{d}+L}\right.} \\
& \left.-V_{a^{\prime \prime} a c d}^{L p p}\left(u_{c} u_{d} v_{a} u_{a^{\prime \prime}}-v_{c} v_{d} u_{a} v_{a^{\prime \prime}}\right)\right](-1)^{j_{a}-j_{a^{\prime \prime}}+L} \frac{\hat{j_{a}}}{\hat{L}} \\
\equiv & \widetilde{V}\left(c d L a ; a^{\prime \prime}\right)(-1)^{j_{a}-j_{a^{\prime \prime}}+L} \frac{\hat{j}_{a}}{\hat{L}} .
\end{aligned}
$$

The $X$ and $Y$ can also be written in the angular momentum coupled form,

$$
\begin{aligned}
X_{c d} & =\sum_{L^{\prime} M^{\prime}}\left\langle j_{c} m_{c} j_{d} m_{d} \mid L^{\prime} M^{\prime}\right\rangle X_{c d}^{L^{\prime} M^{\prime}}, \\
Y_{c d} & =\sum_{L^{\prime} M^{\prime}}\left\langle j_{c} m_{c} j_{d} m_{d} \mid L^{\prime}-M^{\prime}\right\rangle(-1)^{L^{\prime}-M^{\prime}} Y_{c d}^{L^{\prime} M^{\prime}} .
\end{aligned}
$$

So finally $\left\langle a^{\prime \prime}, n L|V| a\right\rangle$ in the angular momentum coupled form is

$$
\begin{aligned}
\left\langle a^{\prime \prime}, n L|V| a\right\rangle= & \left\langle a|V| a^{\prime \prime}, n L\right\rangle=\frac{1}{\sqrt{1+\delta_{c d}}} \sum_{c \geqslant d}\left[V\left(a a^{\prime \prime} c d\right) X_{c d}+V\left(a^{\prime \prime} a c d\right) Y_{c d}\right] \\
= & \frac{1}{\sqrt{1+\delta_{c d}}}\left[\sum_{L^{\prime} M^{\prime}} \sum_{c_{c} j_{d}}\left\langle j_{a} m_{a} j_{a^{\prime \prime}}-m_{a^{\prime \prime}} \mid L M\right\rangle(-1)^{j_{a^{\prime \prime}}-m_{a^{\prime \prime}}} \hat{\hat{L}} \frac{\hat{j_{a}}}{} V\left(c d L a^{\prime \prime} ; a\right) \delta_{L L^{\prime}} \delta_{M M^{\prime}} X_{c d}^{L M}\right. \\
& \left.\times \sum_{L^{\prime} M^{\prime} j_{c} j_{d}}(-1)^{L-M}\left\langle j_{a} m_{a} j_{a^{\prime \prime}}-m_{a^{\prime \prime}} \mid L M\right\rangle(-1)^{j_{a^{\prime \prime}}-m_{a^{\prime \prime}}} \frac{\hat{L}}{\hat{j_{a}}} V\left(c d L a ; a^{\prime \prime}\right) \delta_{L L^{\prime}} \delta_{M M^{\prime}} Y_{c d}^{L M}\right] \\
= & \frac{1}{\sqrt{1+\delta_{c d}}} \sum_{j_{c} j_{d}}\left\langle j_{a} m_{a} j_{a^{\prime \prime}}-m_{a^{\prime \prime}} \mid L M\right\rangle(-1)^{j_{a^{\prime \prime}}-m_{a^{\prime \prime}}}\left[\widetilde{V}\left(c d L a^{\prime \prime} ; a\right) X_{c d}^{L M}+(-1)^{j_{a}-j_{a^{\prime \prime}}+L} \widetilde{V}\left(c d L a ; a^{\prime \prime}\right) Y_{c d}^{L M}\right] \\
\equiv & \left\langle j_{a} m_{a} j_{a^{\prime \prime}}-m_{a^{\prime \prime}} \mid L M\right\rangle(-1)^{j_{a^{\prime \prime}}-m_{a^{\prime \prime}}}\left\langle a^{\prime \prime}, n L\|V\| a\right\rangle \\
= & \left\langle j_{a} m_{a} j_{a^{\prime \prime}}-m_{a^{\prime \prime}} \mid L M\right\rangle(-1)^{j_{a^{\prime \prime}}-m_{a^{\prime \prime}}}\left\langle a\|V\| a^{\prime \prime}, n L\right\rangle .
\end{aligned}
$$

With the above expressions, we can obtain the angular momentum coupled form of $\langle a b|V| N\rangle$, and hence the $W_{a b, a^{\prime} b^{\prime}}^{\downarrow}$. Through the relation

$$
W_{a b a^{\prime} b^{\prime}}^{\downarrow J}=\sum_{m_{a} m_{b} m_{a^{\prime}} m_{b^{\prime}}}\left\langle j_{a} m_{a} j_{b} m_{b} \mid J M_{J}\right\rangle\left\langle j_{a^{\prime}} m_{a^{\prime}} j_{b^{\prime}} m_{b^{\prime}} \mid J M_{J}\right\rangle W_{a b a^{\prime} b^{\prime}}^{\downarrow},
$$

the angular momentum coupled $W_{a b, a^{\prime} b^{\prime}}^{\downarrow J}$ in Eq. (16) will be obtained. 
[1] F. Osterfeld, Rev. Mod. Phys. 64, 491 (1992).

[2] M. Ichimura, H. Sakai, and T. Wakasa, Prog. Part. Nucl. Phys. 56, 446 (2006).

[3] K. Langanke and G. Martínez-Pinedo, Rev. Mod. Phys. 75, 819 (2003).

[4] H.-T. Janka, K. Langanke, A. Marek, G. Martínez-Pinedo, and B. Müller, Phys. Rep. 442, 38 (2007).

[5] E. M. Burbidge, G. R. Burbidge, W. A. Fowler, and F. Hoyle, Rev. Mod. Phys. 29, 547 (1957).

[6] Y.-Z. Qian and G. J. Wasserburg, Phys. Rep. 442, 237 (2007).

[7] Z. M. Niu, Y. F. Niu, H. Z. Liang, W. H. Long, T. Nikšić, D. Vretenar, and J. Meng, Phys. Lett. B 723, 172 (2013).

[8] N. Paar, G. Colò, E. Khan, and D. Vretenar, Phys. Rev. C 80, 055801 (2009).

[9] Y. F. Niu, N. Paar, D. Vretenar, and J. Meng, Phys. Rev. C 83, 045807 (2011).

[10] A. F. Fantina, E. Khan, G. Colò, N. Paar, and D. Vretenar, Phys. Rev. C 86, 035805 (2012).

[11] F. T. Avignone, S. R. Elliott, and J. Engel, Rev. Mod. Phys. 80, 481 (2008).

[12] J. D. Vergados, H. Ejiri, and F. Šimkovic, Rep. Prog. Phys. 75, 106301 (2012).

[13] S. E. Koonin, D. J. Dean, and K. Langanke, Phys. Rep. 278, 1 (1997).

[14] E. Caurier, G. Martìnez-Pinedo, F. Nowacki, A. Poves, and A. P. Zuker, Rev. Mod. Phys. 77, 427 (2005).

[15] S. Fracasso and G. Colò, Phys. Rev. C 72, 064310 (2005).

[16] S. Fracasso and G. Colò, Phys. Rev. C 76, 044307 (2007).

[17] J. Li, G. Colò, and J. Meng, Phys. Rev. C 78, 064304 (2008).

[18] C. L. Bai, H. Sagawa, G. Colò, Y. Fujita, H. Q. Zhang, X. Z. Zhang, and F. R. Xu, Phys. Rev. C 90, 054335 (2014).

[19] M. Bender, J. Dobaczewski, J. Engel, and W. Nazarewicz, Phys. Rev. C 65, 054322 (2002).

[20] P. Sarriguren, Phys. Rev. C 91, 044304 (2015).

[21] K. Yoshida and N. V. Giai, Phys. Rev. C 78, 064316 (2008).

[22] N. Paar, T. Nikšić, D. Vretenar, and P. Ring, Phys. Rev. C 69, 054303 (2004).

[23] N. Paar, D. Vretenar, E. Khan, and G. Colò, Rep. Prog. Phys. 70, 691 (2007).

[24] P. F. Bortignon, R. A. Broglia, D. R. Bes, and R. Liotta, Phys. Rep. 30, 305 (1977).

[25] G. F. Bertsch, P. F. Bortignon, and R. A. Broglia, Rev. Mod. Phys. 55, 287 (1983).

[26] G. Colò, N. Van Giai, P. F. Bortignon, and R. A. Broglia, Phys. Rev. C 50, 1496 (1994).

[27] T. Marketin, E. Litvinova, D. Vretenar, and P. Ring, Phys. Lett. B 706, 477 (2012).

[28] E. Litvinova, B. A. Brown, D.-L. Fang, T. Marketin, and R. G. T. Zegers, Phys. Lett. B 730, 307 (2014).
[29] Y. F. Niu, G. Colò, M. Brenna, P. F. Bortignon, and J. Meng, Phys. Rev. C 85, 034314 (2012).

[30] Y. F. Niu, G. Colò, and E. Vigezzi, Phys. Rev. C 90, 054328 (2014).

[31] Y. F. Niu, Z. M. Niu, G. Colò, and E. Vigezzi, Phys. Rev. Lett. 114, 142501 (2015).

[32] A. Idini, F. Barranco, and E. Vigezzi, Phys. Rev. C 85, 014331(R) (2012).

[33] A. Idini, G. Potel, F. Barranco, E. Vigezzi, and R. A. Broglia, Phys. Rev. C 92, 031304(R) (2015).

[34] Y. Fujita, H. Fujita, T. Adachi, C. L. Bai, A. Algora, G. P. A. Berg, P. von Brentano, G. Colò, M. Csatlós, J. M. Deaven et al., Phys. Rev. Lett. 112, 112502 (2014).

[35] H. Sagawa, C. L. Bai, and G. Colò, Phys. Scr. 91, 083011 (2016).

[36] E. Litvinova, P. Ring, and V. Tselyaev, Phys. Rev. C 78, 014312 (2008).

[37] C. Robin and E. Litvinova, Eur. Phys. J. A 52, 1 (2016).

[38] C. Bai, H. Sagawa, M. Sasano, T. Uesaka, K. Hagino, H. Zhang, X. Zhang, and F. Xu, Phys. Lett. B 719, 116 (2013).

[39] J. Engel, M. Bender, J. Dobaczewski, W. Nazarewicz, and R. Surman, Phys. Rev. C 60, 014302 (1999).

[40] Z. M. Niu, Y. F. Niu, Q. Liu, H. Z. Liang, and J. Y. Guo, Phys. Rev. C 87, 051303 (2013).

[41] Z. Y. Wang, Y. F. Niu, Z. M. Niu, and J. Y. Guo, J. Phys. G: Nucl. Part. Phys. 43, 045108 (2016).

[42] P. Ring and P. Schuck, The Nuclear Many-Body Problem (Springer-Verlag, Berlin, 1980).

[43] J. Bartel, P. Quentin, M. Brack, C. Guet, and H.-B. Håkansson, Nucl. Phys. A 386, 79 (1982).

[44] N. Van Giai and H. Sagawa, Phys. Lett. B 106, 379 (1981).

[45] X. Roca-Maza, G. Colò, and H. Sagawa, Phys. Rev. C 86, 031306 (2012).

[46] V. V. der Sluys, D. V. Neck, M. Waroquier, and J. Ryckebusch, Nucl. Phys. A 551, 210 (1993).

[47] K. Bennaceur and J. Dobaczewski, Comput. Phys. Commun. 168, 96 (2005).

[48] K. Moghrabi, M. Grasso, G. Colò, and N. Van Giai, Phys. Rev. Lett. 105, 262501 (2010).

[49] V. I. Tselyaev, Phys. Rev. C 75, 024306 (2007).

[50] V. I. Tselyaev, Phys. Rev. C 88, 054301 (2013).

[51] D. Gambacurta, M. Grasso, and J. Engel, Phys. Rev. C 92, 034303 (2015).

[52] http://www.nndc.bnl.gov.

[53] K. Pham, J. Jänecke, D. A. Roberts, M. N. Harakeh, G. P. A. Berg, S. Chang, J. Liu, E. J. Stephenson, B. F. Davis, H. Akimune et al., Phys. Rev. C 51, 526 (1995).

[54] M. Sasano, H. Sakai, K. Yako, T. Wakasa, S. Asaji, K. Fujita, Y. Fujita, M. B. Greenfield, Y. Hagihara, K. Hatanaka et al., Phys. Rev. C 79, 024602 (2009). 